\title{
1 Dissecting the collateral damage of antibiotics on gut microbes
}

2 Lisa Maier ${ }^{1,7 \#^{*}}$, Camille V. Goemans ${ }^{1 \#}$, Mihaela Pruteanu ${ }^{1,8}$, Jakob Wirbel ${ }^{2}$, Michael Kuhn ${ }^{2}$

3 Elisabetta Cacace $^{1}$, Tisya Banerjee ${ }^{1}$, Exene Erin Anderson ${ }^{1,9}$, Alessio $^{\text {Milanese }}{ }^{2}$, Ulrike

4 Löber $^{3,4}$, Sofia K. Forslund ${ }^{2,3,4}$, Kiran Raosaheb Patil ${ }^{2}$, Georg Zeller ${ }^{2}$, Peer Bork ${ }^{2,4,5,6}$ and

5 Athanasios Typas ${ }^{1,2^{*}}$

6

$7 \quad$ \# These authors contributed equally to this work

8 * Correspondence: typas@embl.de and I.maier@uni-tuebingen.de

9

$10{ }^{1}$ European Molecular Biology Laboratory, Genome Biology Unit, Heidelberg, Germany

$11{ }^{2}$ European Molecular Biology Laboratory, Structural and Computational Biology Unit,

12 Heidelberg, Germany

$13{ }^{3}$ Experimental and Clinical Research Center, a cooperation of Charité - Universitätsmedizin

14 Berlin and Max Delbruck Center for Molecular Medicine, Berlin, Germany

$15{ }^{4}$ Max-Delbrück-Center for Molecular Medicine, Berlin, Germany

$16{ }^{5}$ Molecular Medicine Partnership Unit, Heidelberg, Germany

$17{ }^{6}$ Department of Bioinformatics, Biocenter, University of Würzburg, Germany

$18{ }^{7}$ current address: Interfaculty Institute of Microbiology \& Infection Medicine Tübingen,

19 Germany

$20{ }^{8}$ current address: Institute for Biology, Humboldt University Berlin, Germany

$21{ }^{9}$ current address: NYU School of Medicine, New York, USA 


\section{Abstract}

Antibiotics are used for fighting pathogens, but also target our commensal bacteria as a side effect, disturbing the gut microbiota composition and causing dysbiosis and disease ${ }^{1-3}$. Despite this well-known collateral damage, the activity spectrum of the different antibiotic classes on gut bacteria remains poorly characterized. Having monitored the activities of $>1,000$ marketed drugs on 38 representative species of the healthy human gut microbiome ${ }^{4}$, we here characterize further the 144 antibiotics therein, representing all major classes. We determined >800 Minimal Inhibitory Concentrations (MICs) and extended the antibiotic profiling to 10 additional species to validate these results and link to available data on antibiotic breakpoints for gut microbes. Antibiotic classes exhibited distinct inhibition spectra, including generation-dependent effects by quinolones and phylogeny-independence by $\beta$ lactams. Macrolides and tetracyclines, two prototypic classes of bacteriostatic protein synthesis inhibitors, inhibited almost all commensals tested. We established that both kill different subsets of prevalent commensal bacteria, and cause cell lysis in specific cases. This species-specific activity challenges the long-standing divide of antibiotics into bactericidal and bacteriostatic, and provides a possible explanation for the strong impact of macrolides on the gut microbiota composition in animals ${ }^{5-8}$ and humans ${ }^{9-11}$. To mitigate the collateral damage of macrolides and tetracyclines on gut commensals, we exploited the fact that drug combinations have species-specific outcomes in bacteria ${ }^{12}$ and sought marketed drugs, which could antagonize the activity of these antibiotics in abundant gut commensal species. By screening $>1,000$ drugs, we identified several such antidotes capable of protecting gut species from these antibiotics without compromising their activity against relevant pathogens. Altogether, this study broadens our understanding of antibiotic action on gut commensals, uncovers a previously unappreciated and broad bactericidal effect of prototypical bacteriostatic antibiotics on gut bacteria, and opens avenues for preventing the collateral damage caused by antibiotics on human gut commensals. 


\section{MAIN TEXT}

Medication is emerging as major contributor for changes in the composition of the human gut microbiota $^{4,13-15}$. Such severe and long-lasting changes are associated, and in some cases causatively linked, to dysbiosis and a wide range of diseases ${ }^{16}$. Although several nonantibiotic drugs may also have a previously unappreciated impact on the gut microbiome composition ${ }^{4,16,17}$, antibiotics, developed to have broad spectra and thereby target very diverse pathogens, are long known to take a heavy toll on our gut flora, causing a variety of gastrointestinal side-effects ${ }^{18}$, including Clostridioides (former Clostridium) difficile infections. Recently more attention has been given to this collateral damage of antibiotics on the gut microbiota and thereby on the host's wellbeing. In vivo studies highlight links between the long-term microbiota compositional changes and host dysbiosis, including the development of allergic, metabolic, immunological and inflammatory diseases ${ }^{5-8,10,11,19-21}$. While uncovering the direct effects of different antibiotics on our gut flora is critical to improve general health, technical difficulties hamper routine testing of antibiotic susceptibility in anaerobes ${ }^{22,23}$. Currently available data on bacterial susceptibility to antibiotics is focused on invasive pathogens and offers little to no resolution in the diversity of the human gut microbiota ${ }^{24}$. Information is missing even for the most prevalent and abundant gut species, or ones recently associated with dysbiosis and disease ${ }^{25,26}$. In addition, existing animal or cohort studies have used a handful of antibiotics or merge data from different antibiotic classes, precluding systematic and general conclusions on the matter.

We recently assessed the direct effect of $~ 1200$ FDA-approved drugs on the growth of 38 prevalent and abundant or disease-associated human gut species under anaerobic conditions at a fixed concentration of $20 \mu \mathrm{M}^{4}$. This initial screen (referred to hereafter as "screen") included 144 antibiotics (Fig. 1a, Extended Data Fig. 1, Suppl. Table 1), with different classes having discernible effects on gut microbes (Fig. 1b). We validated these results by measuring 815 MICs (33 antibiotics and 2 antifungals for 17 species, 22 antibiotics for 10 additional species), using MIC gradient test strips (Fig. 1a, Extended Data Fig. 2, Suppl. Table $2+3$ ). Despite differences in the experimental procedure, concordance 
between data from the initial screen and MICs is very high: Specificity and sensitivity of 0.97 (Extended Data Fig. 3a). The newly established MICs also correlate well with available data on antimicrobial susceptibility from databases such as EUCAST ${ }^{24}$ or $\mathrm{ChEMBL}^{27}\left(\mathrm{r}_{\mathrm{s}}=0.69\right.$ and $r_{s}=0.64$, respectively), despite differences in strains and media used (Extended Data Fig. 3b). Importantly, this new dataset considerably expands the available MICs, as much as by $80 \%$ for non-pathogenic bacteria (Fig. 1c, Extended Data Fig. 3c). Altogether, the initial screen and the new MIC dataset provide high-resolution information on the target spectrum of antibiotics on commensal gut microbes, which we explored further.

The antibiotics tested exhibited strong class-dependent effects (Fig. 1b, d). Consistent with literature, aminoglycosides hardly affected gut microbes under anaerobic conditions $^{28}$ and sulfonamides were inactive in the medium used for the screen ${ }^{4}$. Quinolones acted in a generation-dependent manner. First-generation variants were effective only on a narrow spectrum of microbes that included both commensal E. coli tested. Second- and third- generation quinolones increased the spectrum. Fourth-generation variants (developed to increase activity against anaerobes) inhibited all tested species, except for Akkermansia muciniphila (Fig. 1e, Extended Data Fig. 1, red box), a species associated with protection against different diseases and dysbiotic states ${ }^{29}$, and even positive responses to immunotherapy ${ }^{30}$. For $\beta$-lactams, resistance was patchy but distinct for different members and subclasses (Extended Data Fig. 2, 4a). For Bacteroidetes, we tested additional species and strains (in total 12 and 19, respectively) (Extended Data Fig. 4b, c), confirming that $\beta$ lactam sensitivity and phylogenetic relatedness are uncoupled (Extended Data Fig. 4d). This argues for resistance mechanisms being strain-specific and horizontally transferred. Macrolides showed a strong impact on gut commensals and inhibited all tested microbes (Fig. 1d), except for the opportunistic pathogen C. difficile, which was resistant to all tested macrolides and clindamycin (Extended data Fig. 2, red box). This is in line with the associated risk of $C$. difficile infection after macrolide/clindamycin treatment ${ }^{31}$. Finally, 8 of the 9 tested tetracyclines inhibited nearly all tested microbes, which is surprising in the light of the gut microbiota being considered as reservoir for tetracycline resistance genes ${ }^{32}$. 
Concentration-resolved MICs confirmed the same drug class-dependent trends observed in

107

108

109

110

111 the screen (Fig. 1d, f). In addition, MICs allow for comparisons with clinical breakpoints, i.e. MICs at which a species should be considered resistant or susceptible (Fig. 1f). Overall, the gut microbes in our assays (anaerobic growth, gut mimetic growth medium ${ }^{33}$ ) were slightly more resistant to most antibiotic classes than previously reported for pathogens (aerobic growth, Mueller-Hinton agar). Tetracyclines were the exception, inhibiting commensals at significantly lower concentrations than what is reported for pathogens (Fig. 1f). Thus, commensals might be considerably less resistant to tetracyclines than previously anticipated and suggested by the detection of tetracycline resistance elements in fecal metagenomes.

Recent in-vivo studies have shown that $\beta$-lactams and macrolides have a strong and long-lasting collateral impact on the gut microbiota composition and thereby on host health ${ }^{5-8}$. As $\beta$-lactams exhibited strain-specific effects (Extended Data Fig. 1, 2, 4) and are known to kill bacteria (bactericidal), they could irrevocably deplete specific members of the gut microbiota, thereby explaining their differential and long-lasting effects on the community composition. On the other hand, macrolides uniformly targeted all tested gut commensals (Fig. 1d) and are textbook bacteriostatic antibiotics, i.e. inhibit bacterial growth, but do not kill (at least at high numbers). In this case, the long-term community composition change is more difficult to rationalize, as all community members are inhibited, but should be able to regrow once drug is removed. Similarly, tetracyclines, another class of bacteriostatic antibiotics that acted on nearly all gut microbes we tested, have known gastro-intestinal sideeffects $^{18}$, which are indicative of gut microbiome dysbiosis. We thus wondered at which level macrolides and tetracyclines exert a differential effect on gut microbes. Although traditionally both clinical use $\mathrm{e}^{34-37}$ and basic research ${ }^{38,39}$ heavily rely on this distinction between bactericidal and bacteriostatic antibiotics, there are reports of drugs changing their killing capacity depending on the organism, drug concentration or medium tested ${ }^{40,41}$ (and increased evidence from meta-analyses that the distinction may have little relevance to clinical practice $\left.{ }^{42,43}\right)$. We therefore hypothesized that this bacteriostatic/bactericidal divide may be less rigid for gut commensals, which are more phylogenetically diverse than the few 
pathogens usually tested, and hence provide a level where the effect of these drug classes on gut microbes becomes differential. various time-points after drug treatment. If, over a significant period of antibiotic treatment (ranging from 5 to 24 hours), the number of colony forming units (CFU)/ml of culture decreases by more than $99.9 \%$, the antibiotic is considered bactericidal ${ }^{40}$. We assessed the survival of 12 abundant gut microbes over a 5-hour treatment of either a macrolide (erythromycin or azithromycin) or a tetracycline (doxycycline) at $5 \times \mathrm{MIC}$ (Fig $2 \mathrm{a}+\mathrm{b}$,

143 Extended Data Fig. 5). About half of the tested species decreased in survival by $>99.9 \%$, pointing to these drugs being bactericidal to several abundant gut microbes. To confirm this

145 further, we tested the viability of $B$. vulgatus and E. coli ED1a upon erythromycin, 146 azithromycin or doxycycline treatments using live/dead staining. Microscopy and flow 147 cytometry assessment of live/dead bacteria corroborated the initial observations (Fig. 2c, 148 Extended Data Fig. 6). As tetracyclines are considered bona-fide bacteriostatic drugs in $E$. 149 coli, we were surprised to see that doxycycline effectively killed the commensal E. coli ED1a 150 (Fig. 2a). We verified that these effects held also in the presence of oxygen (Extended Data 151 Fig. 7a) and confirmed that doxycycline has a stronger bactericidal action on this natural 152 isolate than on the domesticated E. coli K-12 lab strain, BW25113 (Extended Data Fig. 7b). 153 In parallel, we excluded that the differences in killing capacity were confounded by growth 154 rate, growth phase or MIC of the bacterial species tested (Extended Data Fig. 8). We also noticed that $B$. vulgatus and $B$. uniformis cultures decreased density in the presence of erythromycin (Fig. 2d). We confirmed by time-lapse microscopy that this was due to lysis. 157 Erythromycin caused cell shape defects, including blebbing, cytoplasmic shrinkage, and 158 ultimately cell lysis in both B. vulgatus and B. uniformis (Figure 2e, Movies 1-4). Altogether, 159 this selective bactericidal activity of macrolides and tetracyclines on specific gut commensals 160 could provide an explanation for the strong effects these drug classes have on the gut 161 microbiota composition of human individuals. The gut microbes killed from the drug would be 
162

inadvertently removed from community, whereas the ones being only inhibited could recover when the therapy stops.

Knowing that drug combinations often have species-specific outcomes ${ }^{12}$, we reasoned that we could identify drugs that selectively antagonize the effect of antibiotics on gut microbes, while retaining activity against pathogens. Therefore, we screened the Prestwick library to identify antagonizing compounds to erythromycin or doxycycline on two abundant and prevalent gut microbes, B. vulgatus and B. uniformis (Fig. 3a, Extended Data Fig. 9). Of the 19 identified hits (Fig. 3b, Suppl. Table 4), we tested the 14 candidates with the strongest activity in a concentration-dependent manner (Extended Data Fig. 10a). Nine retained antagonistic activity over a broader concentration range, which we confirmed by checkerboard assays (Fig. 3c). The antidotes that showed the strongest antagonisms were the anticoagulant drug dicumarol, and two non-steroidal anti-inflammatory drugs, tolfenamic acid and diflunisal. While dicumarol rescued B. vulgatus from erythromycin and diflunisal from doxycycline, tolfenamic acid was able to protect $B$. vulgatus from both drugs. In addition, these interactions were able to at least partially rescue the killing of $B$. vulgatus by erythromycin and doxycycline (Extended Data Fig. 10b). We then probed two of these drugs for their ability to protect other abundant gut commensals and confirmed that both dicumarol and tolfenamic acid were able to counteract erythromycin on several species (Fig. 3d, Extended Data Fig. 11). In contrast, both drugs did not affect the potency of erythromycin on Staphylococcus aureus, Streptococcus pneumoniae and Enterococcus faecium, pathogens against which erythromycin is active/prescribed (Fig. 3e, Extended Data Fig. 12a). For example, tolfenamic acid and dicumarol at concentration ranges of 5-40 $\mu \mathrm{M}$ could rescue the growth of five out of seven tested abundant gut commensal species at clinically relevant erythromycin concentrations (Fig. 3f, Extended Data Fig. 12b). Altogether, our data provides a proof-of-principle for identifying antidotes that specifically mask the collateral damage of antibiotics on commensals. This concept would need to be further validated in the future in animal models. Antidotes may also need to be modified to late (colon)-release or non- 
absorbable formulations to ensure they reach the gut and to minimize adverse effects from

190 their primary action.

In summary, our study provides a high-resolution map of the collateral damage of antibiotics on 50 different resident gut microbes down to the level of individual drugs, species and partially even strains. We challenge the universal divide of antibiotics into bacteriostatic

194 and bactericidal across bacteria, as this breaks down when tested beyond model organisms.

195 Antibiotics with preferential killing of some species may be the most detrimental to our gut 196 flora, although the first studies in a few healthy individuals point to the gut microbiota having 197 some resilience against specific antibiotic regimens ${ }^{44}$. Understanding the underlying 198 mechanisms for this selective killing might open up ways for the development of new 199 antimicrobials, but also strategies for controlled microbiome modulation ${ }^{15}$. Finally, we provide 200 a proof-of-concept that species-specificity of drug combinations ${ }^{12}$ can be exploited to identify 201 antidotes that selectively protect the gut microbiota from the adverse effects of systemic 202 antibiotic therapy. This new approach adds to proposed and existing strategies of gut 203 microbiota protection against antibiotics, such as co-administration of activated charcoal ${ }^{45}, \beta$ 204 lactamases $^{46}$, probiotics or (autologous) fecal transplants ${ }^{47}$. Overall, our results suggest that 205 interactions of antibiotics and commensals merit deeper exploration, as our current 206 knowledge of the mode(s) of action of antibiotics in model pathogens is not necessarily transferable to commensals. 


\section{METHODS}

\section{Growth conditions}

All experiments from this study were performed in an anaerobic chamber (Coy Laboratory Products Inc) $\left(2 \% \mathrm{H}_{2}, 12 \% \mathrm{CO}_{2}, 86 \% \mathrm{~N}_{2}\right)$ and all materials and solutions used for these experiments were pre-reduced for at least $24 \mathrm{~h}$ before use unless specified otherwise.

213 Bacteria used in this study were typically pre-cultured for two overnights: Cells were cultured 214 in $5 \mathrm{ml}$ modified Gifu Anaerobic Medium broth (MGAM) (HyServe GmbH \& Co.KG, Germany, 215 produced by Nissui Pharmaceuticals) and grown at $37^{\circ} \mathrm{C}$ overnight. The next day, cells were 216 diluted $1 / 100$ in $5 \mathrm{ml}$ MGAM medium and grown at $37^{\circ} \mathrm{C}$ for a second overnight before 217 starting the experiments.

Quantitative assay for minimum inhibitory concentration determination with MICs test strips

MICs test strips were purchased from Liofilchem or Oxoid (Suppl. Table 2). All MICs were measured under anaerobic growth conditions inside a Coy anaerobic chamber. Bacteria were precultured in MGAM for two overnights and cultures were diluted to $\mathrm{OD}_{578}=0.5 .50 \mu \mathrm{l}$ of the diluted culture were spread on a MGAM agar plate and allowed to dry for $15 \mathrm{~min}$. The MIC test strip was placed on the agar with sterile tweezers, allowing the part with the lowest concentration touch the agar first. Plates were incubated at $37^{\circ} \mathrm{C}$ inside the anaerobic chamber, at least overnight and longer depending on the species-specific growth requirements. After formation of a symmetrical inhibition ellipse, plates were taken out of the chamber and imaged under controlled lighting conditions (splmager S\&P Robotics Inc.) using an 18 megapixel Canon Rebel T3i (Canon Inc. USA). MICs were directly determined from

231 the strip scale at the point where the edge of the inhibition ellipse intersects the MIC test strip. All MICs were determined in duplicates. In cases of an eight-fold difference between

233 the two values, a third replicate was done. In all cases, this resulted in a clear outlier (> 8-fold different from other two MICs) that was removed from the dataset. 


\section{MIC comparison to ChEMBL and EUCAST databases}

237 Previously known MICs were extracted from the ChEMBL database (version 24$)^{27}$ and 238 EUCAST (obtained on May 14, 2018) ${ }^{24}$. Antibiotics from these two datasets were mapped to 239 our dataset by name. Species were mapped using NCBI Taxonomy Identifiers and species names. For MICs from ChEMBL, a keyword-based approach was used to exclude experiments on species with mutations, deletions, insertions, etc. The EUCAST database 242 contains a large number of reported MICs for each compound-species pair. We collapsed 243 these to a single value by calculating the median MIC. microbiome were calculated using mOTUs $v 2^{48}$ as follows: Relative species abundances were determined in 727 shotgun metagenomic samples from donors in the control groups of 247 multiple studies from various countries and continents ${ }^{49-53}$. Prior to taxonomic profiling, 248 metagenomes were quality controlled using the MOCAT2 -rtf procedure ${ }^{54}$, which removed 249 reads with $\geq 95 \%$ sequence identity and an alignment length of $\geq 45 \mathrm{bp}$ to the human genome 250 hg19. Taxonomic profiles were then created using mOTUs version $2.1 .0^{48}$ with parameters $-\mathrm{I}$ $25175 ;-\mathrm{g} 2$; and -c. Afterwards relative abundances below $10^{-4}$ were set to zero and species 252 with nonzero abundance in $<5$ samples discarded. For the retained 1,350 species, 253 prevalence was defined as the percentage of samples with nonzero abundance; a 254 prevalence cut-off of $1 \%$ was chosen to classify species into "rare" and "common" species. 255 For all species in the MIC dataset, we manually assessed their status as pathogenic or non256 pathogenic species using encyclopaedic and literature knowledge. Pathogenic species that 257 occur in more than $1 \%$ of healthy people (i.e. are designated as "common") were classified 258 as "potentially pathogenic species" that can, for example, cause diseases in 259 immunocompromised patients.

\section{Killing curves and survival assay}

262 Cells were precultured as described in the growth conditions section before being diluted to 263 an $\mathrm{OD}_{578}=0.01$ and grown for $2 \mathrm{~h}$ at $37^{\circ} \mathrm{C}$ (unless specified otherwise). Next, cells were 

(final antibiotic concentration is 5-fold MIC) and incubated in the presence of the antibiotic for $5 \mathrm{~h}$ at $37^{\circ} \mathrm{C}$. At several time-points $(0,1 \mathrm{~h}, 2 \mathrm{~h}, 3 \mathrm{~h}, 4 \mathrm{~h}, 5 \mathrm{~h}), 100 \mu \mathrm{l}$ of cells were serial-diluted in PBS $\left(10^{-1}\right.$ to $10^{-8}$ dilutions) and plated on MGAM-Agar plates for CFU counting. When no cells were detected using this method, a bigger volume of culture (up to $2 \mathrm{ml}$ ) was plated to be able to detect CFUs. Agar plates were incubated overnight at $37^{\circ} \mathrm{C}$ and colonies were counted the next day, either manually, for low CFU numbers, or using the Analyze Particles

271 tool from Image $J^{55}$.

\section{Live/dead staining}

274 Cells were precultured as described in the growth conditions section before being diluted to 275 an $\mathrm{OD}_{578}=0.01$ and grown for $2 \mathrm{~h}$ at $37^{\circ} \mathrm{C}$. Cells were next diluted $1 / 2$ in MGAM containing 10-fold MIC of erythromycin, azithromycin or doxycycline (final concentration is 5-fold the MIC) and incubated in the presence of the antibiotic for $5 \mathrm{~h}$ at $37^{\circ} \mathrm{C}$. Then, cells were live/dead stained using the LIVE/DEAD BacLight Bacterial viability and counting kit (\#L34856 Molecular Probes, ThermoFisher) according to the manufacturer's protocol before and after antibiotic treatment.

\section{Flow cytometry}

283 Stained cells were counted using a BD LSRFortessa ${ }^{T M}$ flow cytometer. The forward and side 284 scatter signals $(488 \mathrm{~nm})$ as well as the green and red fluorescent signals (488-530/30A filter and 561-610/20A filter, respectively) were acquired. The FSC/SSC detectors were set to logarithmic scale. The flow rate varied between $12 \mu \mathrm{l} / \mathrm{min}$ and $60 \mu \mathrm{l} / \mathrm{min}$ depending on the concentration of each sample, and the analysis was stopped when 10,000 target events were measured. Graphs were generated using the FlowJo V10.3 software (Treestar). 
291 For live/dead imaging, stained cells were washed twice in $0.85 \% \mathrm{NaCl}$ before being spotted 292 on $0.85 \% \mathrm{NaCl}+1 \%$ agarose pads between a glass slide and a coverslip. For time-lapse 293 imaging, cells were precultured as described in the growth conditions section. Cells were 294 then diluted to an $\mathrm{OD}_{578}=0.01$ and grown for $3 \mathrm{~h}$ at $37^{\circ} \mathrm{C}$ before being spotted on MGAM $295+1 \%$ agarose pads, supplemented or not with $15 \mu \mathrm{g} / \mathrm{ml}$ erythromycin (5-fold MIC) between a glass slide and a coverslip. Slides were sealed with valap (to avoid/delay oxygen permeation) and taken outside of the anaerobic chamber for imaging. In these conditions, 298 untreated bacteria kept growing rapidly (Movie $1+3)$. The imaging was performed using a 299 Nikon Eclipse Ti inverted microscope, equipped with a Nikon DS-Qi2 camera, a Nikon Plan Apo Lambda 60X oil Ph3 DM phase contrast objective and a Nikon HC mCherry filter set (Ex 562/40; DM 593; BA 641/75) to detect propidium iodide fluorescence. Images were acquired with the NIS-Elements AR4.50.00 software and processed with Fiji v.2.0.0-rc-68/1.52 ${ }^{56}$.

\section{Growth curves}

305 Cells were precultured as described in the growth conditions section. Then, cells were diluted 306 to an $\mathrm{OD}_{578}=0.01$ in a 96 -well plate sealed with a breathable membrane (Breathe-Easy ${ }^{8}$ ) 307 and grown for $2 \mathrm{~h}$. Next, erythromycin was added to the culture to a final concentration of 15 $308 \mu \mathrm{g} / \mathrm{ml}$ (5-fold MIC) and growth curves were acquired for $20 \mathrm{~h}$ using a microplate spectrophotometer (EON, Biotek) by measuring the $\mathrm{OD}_{578}$ every hour after $30 \mathrm{sec}$ of linear shaking.

\section{Screen for microbiome-protective antibiotic antagonism}

313 Preparation of screening plates. The Prestwick Chemical Library was purchased from 314 Prestwick Chemical Inc. and drugs were re-arrayed, diluted and stored in 96 well format as described before ${ }^{4}$. We prepared drug plates ( $2 \times$ drug concentration) in MGAM medium and stored them at $-30^{\circ} \mathrm{C}$. For each experiment, drug plates were thawed, supplemented with the respective antibiotic solution (freshly prepared in MGAM) and pre-reduced in the anaerobic 
chamber overnight. All rearranging and aliquoting steps were done using the Biomek FXP

(Beckman Coulter) system.

Inoculation and screening conditions. Strains were grown twice overnight, the second overnight culture was diluted in MGAM to reach $\mathrm{OD}_{578 \mathrm{~nm}} 0.04$ (4 $\mathrm{x}$ the desired starting OD). $25 \mu \mathrm{l}$ of the diluted cultures were used to inoculate wells containing $50 \mu \mathrm{l}$ of $2 \mathrm{x}$ concentrated Prestwick drug and $25 \mu \mathrm{l}$ of the $4 x$ concentrated antibiotic using the semi-automated, 96-well multi-channel pipette epMotion96 (Eppendorf). Each well contained 1\% DMSO, $20 \mu \mathrm{M}$ of the Prestwick drug and a species-specific antibiotic concentration that was just inhibitory for the respective strain $(0.625 \mu \mathrm{M}$ for erythromycin, $0.04 \mu \mathrm{M}$ doxycycline for $B$. uniformis and 0.08 $\mu \mathrm{M}$ doxycycline for $B$. vulgatus). Plates were sealed with breathable membranes (BreatheEasy $\left.{ }^{\circledR}\right)$ and $\mathrm{OD}_{578}$ was measured hourly after $30 \mathrm{sec}$ of linear shaking with a microplate spectrophotometer (EON, Biotek) and an automated microplate stacker (Biostack 4, Biotek)

330 fitted inside a custom-made incubator (EMBL Mechanical Workshop). Growth curves were 331 collected up to $24 \mathrm{~h}$. For each antibiotic, each species was screen in biological duplicates. All experiments included control wells of unperturbed growth (32 wells per run) and control wells 333 for growth in the presence of the antibiotic only (8 wells per plate).

334 Analysis pipeline and hit calling. All growth curves within a plate were truncated at the transition time from exponential to stationary phase and converted to normalized AUCs using in-run control wells (no drug) as described before ${ }^{4}$.We then calculated z-scores based on

337 these normalized AUCs, removed replicates with 8-fold differences in z-scores to eliminate noise effects, computed mean z- scores across the two replicates and selected combinations with mean z-scores $>3$. This selection included 19 potential antibiotic antagonists and we followed up on 14 of them (7 potential erythromycin and 7 potential doxycycline antagonists 341 in either B. vulgatus or B. uniformis - see Extended Data Fig. 9) in independent experiments. 342 Validation of microbiome-protective antagonists. First, we kept the erythromycin/doxycycline 343 concentration constant $(0.625 \mu \mathrm{M}$ for erythromycin, $0.078 \mu \mathrm{M}$ (B. vulgatus)/ $0.039 \mu \mathrm{M}(B$. 344 uniformis) for doxycycline) and tested concentration gradients of the potential antagonists 345 with ranges depending on the antagonist's solubility. Compounds were purchased from 
independent vendors (Suppl. Table 5) and dissolved at 100x starting concentration in DMSO.

347 Eight 2-fold serial dilutions were prepared in 96-well plates with each row containing a 348 different antagonist, sufficient control DMSO wells and wells with just the respective antibiotic 349 ('antibiotic-only' control). These master plates were diluted in MGAM medium (50 $\mu$ l) to $2 \mathrm{x}$ assay concentration and $25 \mu \mathrm{l}$ freshly prepared antibiotic solution (4x test concentration) was

351 added. Plates were pre-reduced overnight in an anaerobic chamber and inoculated with 25 $352 \mu \mathrm{l}$ of overnight cultures (prepared as described under Growth conditions) to reach a starting $353 \mathrm{OD}_{578}$ of 0.01 and $1 \%$ DMSO concentration. Growth was monitored hourly for $24 \mathrm{~h}$ after 30 354 sec of linear shaking (as described for the screen $^{4}$ ). Experiments were performed in 355 biological triplicates. For analysis, growth curves were converted into normalized AUCs (see above). We accounted for residual growth in the presence of the antibiotic by subtracting the median normalized AUCs of the 'antibiotic-only' control per plate. We computed medians 358 across triplicates and considered a normalized AUC $>0.25$ as concentration-dependent 359 growth rescue by the antagonist.

360 Checkerboard assays for anaerobic commensals. Validated antagonists were further investigated in $8 \times 8$ checkerboard assays, where both antibiotics and antagonists were titrated against each other. Such assays were first performed for the commensals that were originally screened (i. e. B. vulgatus and B. uniformis -4 replicates) and later expanded 364 towards six further gut microbes (B. caccae, B. fragilis NT, B. ovatus, $B$. thetaiotaomicron, $P$. 365 copri, P. distasonis - 2 replicates). For vertical gradients, 2 -fold serial dilutions of the antagonists were prepared first in 100x in DMSO and diluted in MGAM as described above 367 (section 'Validation of microbiome-protective antagonists'). Horizontal antibiotic dilution series were freshly prepared in MGAM at $4 x$ final concentration in equidistant concentration steps. Both, vertical and horizontal dilution series were combined $(50 \mu \mathrm{l}$ of the antagonist gradients $(2 x)$ and $25 \mu l$ of the antibiotic gradients $(4 x))$ in 96 well plates. Plates were pre371 reduced under anaerobic conditions overnight, inoculated with $25 \mu \mathrm{l}$ of diluted overnight 372 culture (at $4 x$ starting OD) and sealed with breathable membrane (Breathe-Easy®). Bacterial 373 growth was monitored once per hour for $24 \mathrm{~h}$ after $30 \mathrm{sec}$ linear shaking (Eon + Biostack 4, 
Biotek) under anaerobic conditions. Growth curves were converted into normalized AUCs as

375 described using in-plate controls to define unperturbed growth.

376 Checkerboard assays for pathogens under aerobic conditions. For three pathogens (S. aureus DSM20231 ATCC 12600 and E. faecium ATCC19434) 8x8 checkerboard assays were performed in transparent 384 well plates (Greiner BioOne $\mathrm{GmbH}$ ), with each well containing a total volume of $30 \mu \mathrm{l}$ in total for S. aureus and $55 \mu \mathrm{l}$ for E. faecium. S. aureus strains were grown in tryptic soy broth (TSB, Sigma Aldrich), E. faecium in BHI medium

381 (Sigma Aldrich). Drugs were arrayed in 2-fold serial dilutions for the checkerboards. Cell 382 were inoculated at initial $\mathrm{OD}_{595 \mathrm{~nm}} \sim 0.01$ from an overnight culture. Plates were sealed with 383 breathable membranes (Breathe-Easy), incubated at $37^{\circ} \mathrm{C}$ (Cytomat 2, Thermo Scientific) 384 with continuous shaking and $\mathrm{OD}_{595 \mathrm{~nm}}$ was measured every 30 min for $16 \mathrm{~h}$ in a Filtermax F5 multimode plate reader (Molecular Devices). For S. pneumoniae D39, we only tested concentration gradients of the potential antagonists in a constant antibiotic concentration $(0.2$ $\mu \mathrm{M}$ erythromycin) in $\mathrm{BHI}$ medium. All experiments were done at least in 2 biological replicates and 2 technical replicates. Wells in which there was significant condensation were removed and background due to medium was subtracted. Growth curves were trimmed at the transition to stationary phase ( $9 \mathrm{~h}$ for $S$. aureus, $12 \mathrm{~h}$ for $E$. faecium). AUCs were calculated and normalised by the median of the internal no-drug control wells $(n=6)$. Interactions were quantified according to the Bliss interaction model ${ }^{57}$. Interactions were called antagonistic if the median of all the interaction scores for a given checkerboard was 394 above 0.05 , synergistic if the value was below -0.05 and neutral if lying between these two 395 cut-offs.

\section{Phylogenetic analysis/phylogenetic tree construction}

398 In order to generate a phylogenetic tree for the different isolates, the nucleotide sequences

399 for a set of universally occurring, protein coding, single copy phylogenetic marker genes ${ }^{48,58}$ 400 were extracted from reference genomes or genome assemblies using fetchMG ${ }^{58}$ 401 (https://motu-tool.org/fetchMG.html). Within the framework of the ete3 toolkit ${ }^{59}$, 
402 ClustalOmega ${ }^{60}$ was used to create sequence alignments for each marker gene

403 independently and all columns with more than $10 \%$ gaps were removed. The individual

404 alignments were concatenated and finally, a phylogenetic tree was inferred from the 405 combined alignment using IQTree ${ }^{61}$.

406

407 Data availability

408 Data is available upon request.

409

410 Code availability

411 Code is available upon request.

412 


\section{REFERENCES}

Blaser, M. J. Antibiotic use and its consequences for the normal microbiome. Science 352, 544-545, doi:10.1126/science.aad9358 (2016).

2 Modi, S. R., Collins, J. J. \& Relman, D. A. Antibiotics and the gut microbiota. J Clin Invest 124, 4212-4218, doi:10.1172/JCI72333 (2014).

3 Becattini, S., Taur, Y. \& Pamer, E. G. Antibiotic-Induced Changes in the Intestinal Microbiota and Disease. Trends Mol Med 22, 458-478, doi:10.1016/j.molmed.2016.04.003 (2016).

4 Maier, L. et al. Extensive impact of non-antibiotic drugs on human gut bacteria. Nature 555, 623-628, doi:10.1038/nature25979 (2018).

5 Cho, l. et al. Antibiotics in early life alter the murine colonic microbiome and adiposity. Nature 488, 621-626, doi:10.1038/nature11400 (2012).

6 Cox, L. M. et al. Altering the intestinal microbiota during a critical developmental window has lasting metabolic consequences. Cell 158, 705-721, doi:10.1016/j.cell.2014.05.052 (2014).

$7 \quad$ Nobel, Y. R. et al. Metabolic and metagenomic outcomes from early-life pulsed antibiotic treatment. Nat Commun 6, 7486, doi:10.1038/ncomms8486 (2015).

8 Ruiz, V. E. et al. A single early-in-life macrolide course has lasting effects on murine microbial network topology and immunity. Nat Commun 8, 518, doi:10.1038/s41467017-00531-6 (2017).

9 Hirsch, A. G. et al. Early-life antibiotic use and subsequent diagnosis of food allergy and allergic diseases. Clin Exp Allergy 47, 236-244, doi:10.1111/cea.12807 (2017).

10 Korpela, K. et al. Intestinal microbiome is related to lifetime antibiotic use in Finnish pre-school children. Nat Commun 7, 10410, doi:10.1038/ncomms10410 (2016).

11 Parker, E. P. K. et al. Changes in the intestinal microbiota following the administration of azithromycin in a randomised placebo-controlled trial among infants in south India. Sci Rep 7, 9168, doi:10.1038/s41598-017-06862-0 (2017).

12 Brochado, A. R. et al. Species-specific activity of antibacterial drug combinations. Nature 559, 259-263, doi:10.1038/s41586-018-0278-9 (2018).

13 Falony, G. et al. Population-level analysis of gut microbiome variation. Science $\mathbf{3 5 2}$, 560-564, doi:10.1126/science.aad3503 (2016).

14 Rothschild, D. et al. Environment dominates over host genetics in shaping human gut microbiota. Nature 555, 210-215, doi:10.1038/nature25973 (2018).

15 Schmidt, T. S. B., Raes, J. \& Bork, P. The Human Gut Microbiome: From Association to Modulation. Cell 172, 1198-1215, doi:10.1016/j.cell.2018.02.044 (2018).

16 Jackson, M. A. et al. Gut microbiota associations with common diseases and prescription medications in a population-based cohort. Nat Commun 9, 2655, doi:10.1038/s41467-018-05184-7 (2018).

17 Maier, L. \& Typas, A. Systematically investigating the impact of medication on the gut microbiome. Curr Opin Microbiol 39, 128-135, doi:10.1016/j.mib.2017.11.001 (2017).

18 Kuhn, M., Letunic, I., Jensen, L. J. \& Bork, P. The SIDER database of drugs and side effects. Nucleic Acids Res 44, D1075-1079, doi:10.1093/nar/gkv1075 (2016).

19 Arrieta, M. C. et al. Early infancy microbial and metabolic alterations affect risk of childhood asthma. Sci Trans/ Med 7, 307ra152, doi:10.1126/scitranslmed.aab2271 (2015).

20 Dethlefsen, L. \& Relman, D. A. Incomplete recovery and individualized responses of the human distal gut microbiota to repeated antibiotic perturbation. Proc Natl Acad Sci U S A 108 Suppl 1, 4554-4561, doi:10.1073/pnas.1000087107 (2011).

21 Saari, A., Virta, L. J., Sankilampi, U., Dunkel, L. \& Saxen, H. Antibiotic exposure in infancy and risk of being overweight in the first 24 months of life. Pediatrics 135, 617626, doi:10.1542/peds.2014-3407 (2015).

22 Gajdacs, M., Spengler, G. \& Urban, E. Identification and Antimicrobial Susceptibility Testing of Anaerobic Bacteria: Rubik's Cube of Clinical Microbiology? Antibiotics (Basel) 6, doi:10.3390/antibiotics6040025 (2017). 
23 Nagy, E., Boyanova, L., Justesen, U. S. \& Infections, E. S. G. o. A. How to isolate, identify and determine antimicrobial susceptibility of anaerobic bacteria in routine laboratories. Clin Microbiol Infect 24, 1139-1148, doi:10.1016/j.cmi.2018.02.008 (2018).

24 The European Committee on Antimicrobial Susceptibility Testing. Breakpoint tables for interpretation of MICs and zone diameters, v., 2019.

http://www.eucast.org/clinical_breakpoints/. .

25 Bullman, S. et al. Analysis of Fusobacterium persistence and antibiotic response in colorectal cancer. Science 358, 1443-1448, doi:10.1126/science.aal5240 (2017).

26 Manfredo Vieira, S. et al. Translocation of a gut pathobiont drives autoimmunity in mice and humans. Science 359, 1156-1161, doi:10.1126/science.aar7201 (2018).

27 Gaulton, A. et al. The ChEMBL database in 2017. Nucleic Acids Res 45, D945-D954, doi:10.1093/nar/gkw1074 (2017).

28 Bryan, L. E., Kowand, S. K. \& Van Den Elzen, H. M. Mechanism of aminoglycoside antibiotic resistance in anaerobic bacteria: Clostridium perfringens and Bacteroides fragilis. Antimicrob Agents Chemother 15, 7-13, doi:10.1128/aac.15.1.7 (1979).

29 Cani, P. D. \& de Vos, W. M. Next-Generation Beneficial Microbes: The Case of Akkermansia muciniphila. Front Microbiol 8, 1765, doi:10.3389/fmicb.2017.01765 (2017).

30 Routy, B. et al. Gut microbiome influences efficacy of PD-1-based immunotherapy against epithelial tumors. Science 359, 91-97, doi:10.1126/science.aan3706 (2018).

31 Slimings, C. \& Riley, T. V. Antibiotics and hospital-acquired Clostridium difficile infection: update of systematic review and meta-analysis. $J$ Antimicrob Chemother 69, 881-891, doi:10.1093/jac/dkt477 (2014).

32 Baron, S., Diene, S. \& Rolain, J.-M. Human microbiomes and antibiotic resistance. Human Microbiome Journal (2018).

33 Tramontano, M. et al. Nutritional preferences of human gut bacteria reveal their metabolic idiosyncrasies. Nat Microbiol 3, 514-522, doi:10.1038/s41564-018-0123-9 (2018).

34 Cohen J, O. S. M., Powderly W G, editors. . Infectious diseases. 2 vol. 3rd ed. St. Louis, Mo.; London: Mosby. (2010.).

35 Habib, G. et al. 2015 ESC Guidelines for the management of infective endocarditis: The Task Force for the Management of Infective Endocarditis of the European Society of Cardiology (ESC). Endorsed by: European Association for Cardio-Thoracic Surgery (EACTS), the European Association of Nuclear Medicine (EANM). Eur Heart J 36, 3075-3128, doi:10.1093/eurheartj/ehv319 (2015).

36 Kasper DL, F. A., Hauser SL, Longo DL. Harrison's Principles of Internal Medicine, 18e, 2012 McGraw-Hill.

37 Liang, S. Y. \& Kumar, A. Empiric antimicrobial therapy in severe sepsis and septic shock: optimizing pathogen clearance. Curr Infect Dis Rep 17, 493, doi:10.1007/s11908-015-0493-6 (2015).

38 Lobritz, M. A. et al. Antibiotic efficacy is linked to bacterial cellular respiration. Proc Natl Acad Sci U S A 112, 8173-8180, doi:10.1073/pnas.1509743112 (2015).

39 Ocampo, P. S. et al. Antagonism between bacteriostatic and bactericidal antibiotics is prevalent. Antimicrob Agents Chemother 58, 4573-4582, doi:10.1128/AAC.02463-14 (2014).

40 French, G. L. Bactericidal agents in the treatment of MRSA infections--the potential role of daptomycin. J Antimicrob Chemother 58, 1107-1117, doi:10.1093/jac/dkl393 (2006).

41 Jelic, D. \& Antolovic, R. From Erythromycin to Azithromycin and New Potential Ribosome-Binding Antimicrobials. Antibiotics (Basel) 5, doi:10.3390/antibiotics5030029 (2016).

42 Nemeth, J., Oesch, G. \& Kuster, S. P. Bacteriostatic versus bactericidal antibiotics for patients with serious bacterial infections: systematic review and meta-analysis. $J$ Antimicrob Chemother 70, 382-395, doi:10.1093/jac/dku379 (2015). 
$52343 \quad$ Wald-Dickler, N., Holtom, P. \& Spellberg, B. Busting the Myth of "Static vs Cidal": A

44 Palleja, A. et al. Recovery of gut microbiota of healthy adults following antibiotic Systemic Literature Review. Clin Infect Dis 66, 1470-1474, doi:10.1093/cid/cix1127 (2018). exposure. Nat Microbiol 3, 1255-1265, doi:10.1038/s41564-018-0257-9 (2018).

de Gunzburg, J. et al. Protection of the Human Gut Microbiome From Antibiotics. The Journal of Infectious Diseases 217, 628-636, doi:10.1093/infdis/jix604 (2017).

46 Stiefel, U., Nerandzic, M. M., Koski, P. \& Donskey, C. J. Orally administered betalactamase enzymes represent a novel strategy to prevent colonization by Clostridium difficile. J Antimicrob Chemother 62, 1105-1108, doi:10.1093/jac/dkn298 (2008).

47 Suez, J. et al. Post-Antibiotic Gut Mucosal Microbiome Reconstitution Is Impaired by Probiotics and Improved by Autologous FMT. Cell 174, 1406-1423 e1416, doi:10.1016/j.cell.2018.08.047 (2018).

48 Milanese, A. et al. Microbial abundance, activity and population genomic profiling with mOTUs2. Nat Commun 10, 1014, doi:10.1038/s41467-019-08844-4 (2019).

49 Feng, Q. et al. Gut microbiome development along the colorectal adenomacarcinoma sequence. Nat Commun 6, 6528, doi:10.1038/ncomms7528 (2015).

50 Vogtmann, E. et al. Colorectal Cancer and the Human Gut Microbiome: Reproducibility with Whole-Genome Shotgun Sequencing. PLoS One 11, e0155362, doi:10.1371/journal.pone.0155362 (2016).

51 Wirbel, J. et al. Meta-analysis of fecal metagenomes reveals global microbial signatures that are specific for colorectal cancer. Nat Med 25, 679-689, doi:10.1038/s41591-019-0406-6 (2019).

$52 \mathrm{Yu}, \mathrm{J}$. et al. Metagenomic analysis of faecal microbiome as a tool towards targeted non-invasive biomarkers for colorectal cancer. Gut 66, 70-78, doi:10.1136/gutjnl2015-309800 (2017).

53 Zeller, G. et al. Potential of fecal microbiota for early-stage detection of colorectal cancer. Mol Syst Biol 10, 766, doi:10.15252/msb.20145645 (2014).

54 Kultima, J. R. et al. MOCAT2: a metagenomic assembly, annotation and profiling framework. Bioinformatics 32, 2520-2523, doi:10.1093/bioinformatics/btw183 (2016).

55 Schneider, C. A., Rasband, W. S. \& Eliceiri, K. W. NIH Image to ImageJ: 25 years of image analysis. Nat Methods 9, 671-675 (2012).

56 Schindelin, J. et al. Fiji: an open-source platform for biological-image analysis. Nat Methods 9, 676-682, doi:10.1038/nmeth.2019 (2012).

$57 \mathrm{Cl}$, B. The toxicity of poisons applied jointly. Ann Appl Biol 26, 585-615 (1939).

58 Sunagawa, S. et al. Metagenomic species profiling using universal phylogenetic marker genes. Nat Methods 10, 1196-1199, doi:10.1038/nmeth.2693 (2013).

59 Huerta-Cepas, J., Serra, F. \& Bork, P. ETE 3: Reconstruction, Analysis, and Visualization of Phylogenomic Data. Mol Biol Evol 33, 1635-1638, doi:10.1093/molbev/msw046 (2016).

60 Sievers, F. et al. Fast, scalable generation of high-quality protein multiple sequence alignments using Clustal Omega. Mol Syst Biol 7, 539, doi:10.1038/msb.2011.75 (2011).

61 Nguyen, L. T., Schmidt, H. A., von Haeseler, A. \& Minh, B. Q. IQ-TREE: a fast and effective stochastic algorithm for estimating maximum-likelihood phylogenies. Mol Biol Evol 32, 268-274, doi:10.1093/molbev/msu300 (2015). 


\section{SUPPLEMENTARY INFORMATION}

\section{Supplementary tables}

\section{Supplementary Table 1}

575 Annotation and classification of antibiotics

\section{Supplementary Table 2}

577 MICs strips used in this study

\section{Supplementary Table 3}

579 MICs as determined by MIC strips for selected antibiotics

\section{Supplementary Table 4}

581 Hitlist: Screen for microbiome-protective antagonists to erythromycin/tetracycline

582 Supplementary Table 5

583 Compounds used in this study

\section{Supplementary movies}

585 Movie 1

586 Time-lapse of $B$. vulgatus growing on MGAM-Agarose 1\% pad.

\section{Movie 2}

588 Time-lapse of B. vulgatus growing on MGAM-Agarose $1 \%$ pad containing 5 -fold MIC of

589 erythromycin.

\section{$590 \quad$ Movie 3}

591 Time-lapse of $B$. uniformis growing on MGAM-Agarose $1 \%$ pad.

\section{Movie 4}

593 Time-lapse of $B$. uniformis growing on MGAM-Agarose 1\% pad containing 5-fold MIC of 594 erythromycin.

\section{Acknowledgements}

597 We thank Stephan Göttig and the Typas lab for feedback on the manuscript. We thank Ana

598 Rita Brochado, Sarela Santamarina and the EMBL Flow Cytometry Core Facility for help with

599 experimental design. We acknowledge EMBL and the JPIAMR grant Combinatorials for 
600 funding. LM and MP were supported by the EMBL Interdisciplinary Postdoc (EIPOD)

601 program under Marie Sklodowska Curie Actions COFUND (grant 291772) and LM is now

602 supported by the CMFI Cluster of Excellence (EXC 2124). CG is recipient of an EMBO long-

603 term postdoctoral fellowship and an add-on fellowship from the Christiane Nüsslein-Volhard-

604 Stiftung. AT is supported by ERC consolidator grant UCARE.

605

606

607

\section{Author contributions}

608 This study was conceived by KRP, PB and AT; designed by LM, CVG, MP and AT; and 609 supervised by LM and AT. Experiments were conducted by LM, CVG and MP. TB and EEA 610 contributed to MIC measurements and EC to checkerboard analyses. Data preprocessing, 611 curation and comparisons to existing databases were performed by JW, MK, AM, UL, SKF 612 and GZ. Data interpretation was performed by LM, CVG, JW, GZ and AT. LM, CVG and AT 613 wrote the manuscript with feedback from all authors; LM, CVG, JW and MK designed figures 614 with inputs from GZ and AT. All authors approved the final version for publication.

\section{Author information}

617 EMBL has filed a patent application on using the antidotes identified in this study for 618 prevention and/or treatment of dysbiosis and for microbiome protection (European patent 619 application number EP19216548.8). LM, CVG, EC and AT are listed as inventors.

620 Correspondence and requests for materials should be addressed to typas@embl.de or 621 I.maier@uni-tuebingen.de. 
bioRxiv preprint doi: https://doi.org/10.1101/2020.01.09.893560; this version posted January 9, 2020. The copyright holder for this preprint (which was not certified by peer review) is the author/funder, who has granted bioRxiv a license to display the preprint in perpetuity. It is

Screen

144 ABX x 40 species/strains

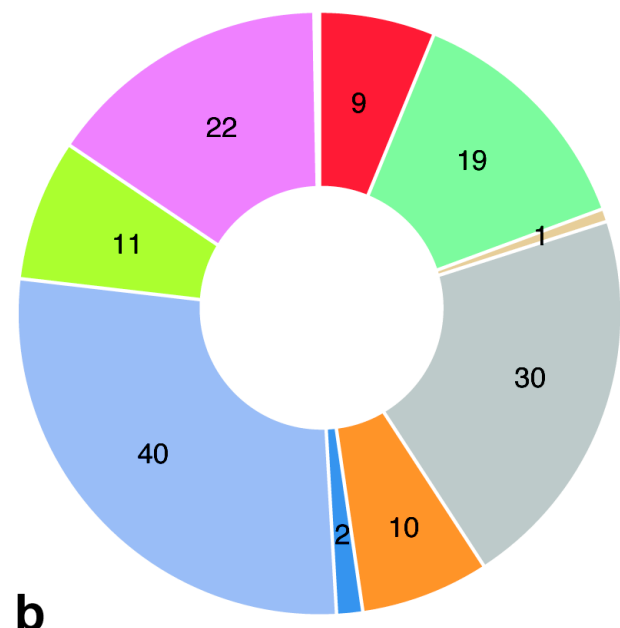

Main classes - Screen

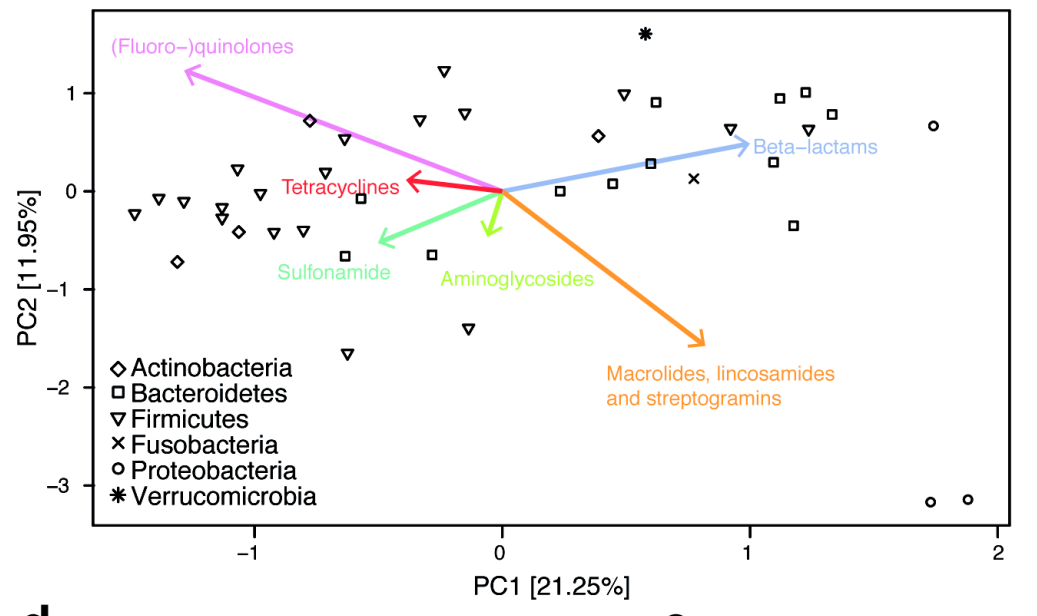

d

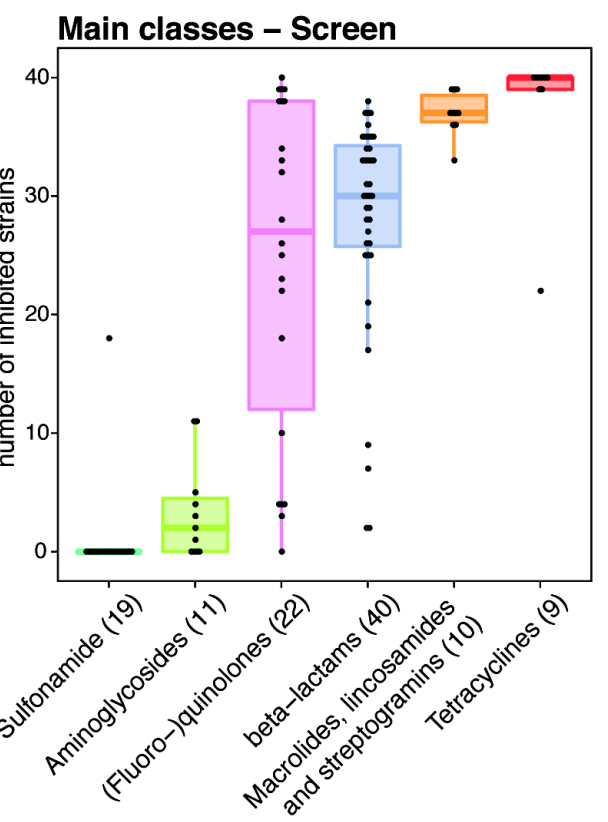

$33 \mathrm{ABX} \times 17$ species

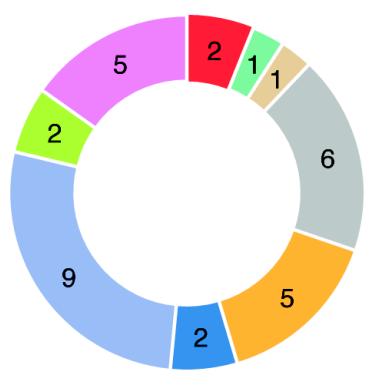

C

e

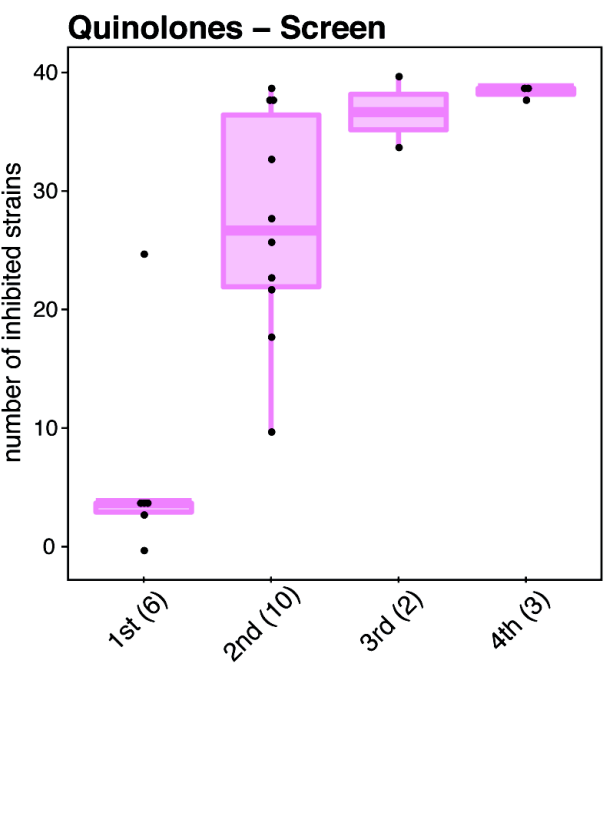

\section{Antibiotic classes}

(Fluoro-)quinolones

Aminoglycosides

beta-lactams

Glycopeptides \& lipoglycopeptides

Macrolides, lincosamides \& streptogramins

Miscellaneous agents

Oxazolidinone

Sulfonamides

Tetracyclines

\section{MICs}

rare nonpathogenic common nonpathogenic pathogenic potentially pathogenic
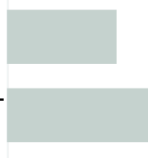

$+80 \%$ new MICs

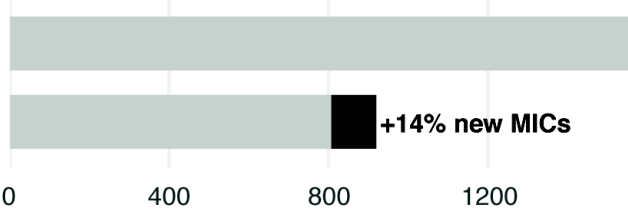

Number of MICs (drug-species pairs)

MICs exist in EUCAST or ChEMBL

New MICs

Figure 1 -Activity spectrum of antibiotic classes on human gut commensals.

a. Overview of antibiotics tested in initial screen at $20 \mu \mathrm{M}$ concentration ${ }^{4}$ and validated by MIC determination in this study. b. Principal component analysis based on AUCs from the initial screen on the effects of antibiotics on gut commensals. Antibiotic classes drive some separation at the phylum level, e.g. beta-lactams separate Bacteroidetes and macrolides/lincosamides/streptogramins separate Proteobacteria. c. Comparison of MICs from this study to MICs available from public databases. Species are classified as "common" or "rare" if they are present in the gut microbiome of more or less than $1 \%$ of 727 healthy individuals, respectively (see Methods). d. For the main antibiotic classes from the screen, the numbers of inhibited strains are shown ( $N$ as in a). 40 strains tested in total at a $20 \mu \mathrm{M}$ antibiotic concentration. Boxes span the IQR and whiskers extend to the most extreme data points up to a max of 1.5 times the IQR. e. Number of inhibited strains per (fluoro-)quinolone drug generation. Number of tested drugs per generation is indicated in brackets on x-axis labeling. Boxplots as in panel d. f. MICs of drug-species pairs for the main antibiotic classes measured in this study are depicted next to EUCAST clinical (susceptibility) breakpoints for pathogens. Numbers of drug-species pairs (MICs; colored) and of antibiotic per class (EUCAST clinical breakpoints; grey) are shown in brackets. Boxplots as in panel, $\mathbf{d}, \mathbf{y}$-axis is log2 scale. 
bioRxiv preprint doi: https://doi.org/10.1101/2020.01.09.893560; this version posted January 9, 2020. The copyright holder for this preprint (which was not certified by peer review) is the author/funder, who has granted bioRxiv a license to display the preprint in perpetuity. It is made available under aCC-BY 4.0 International license.

a
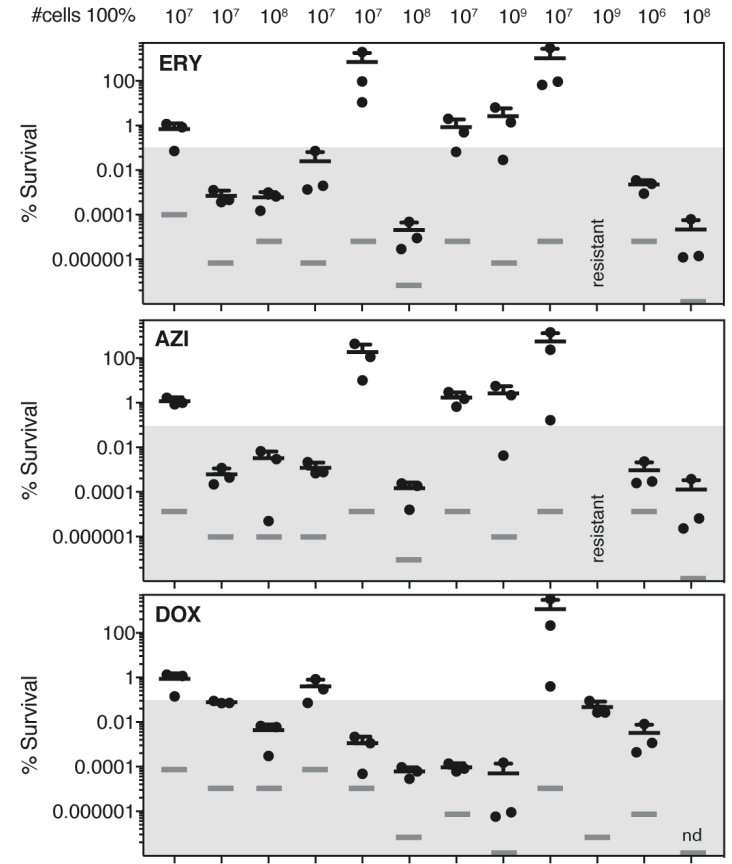

d
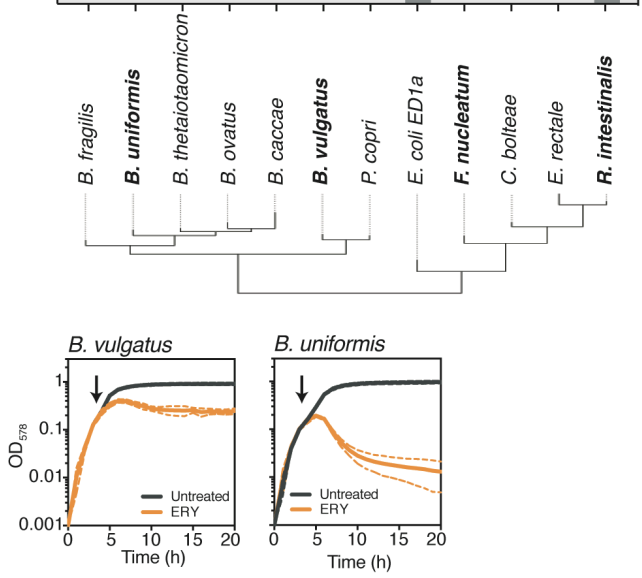

e
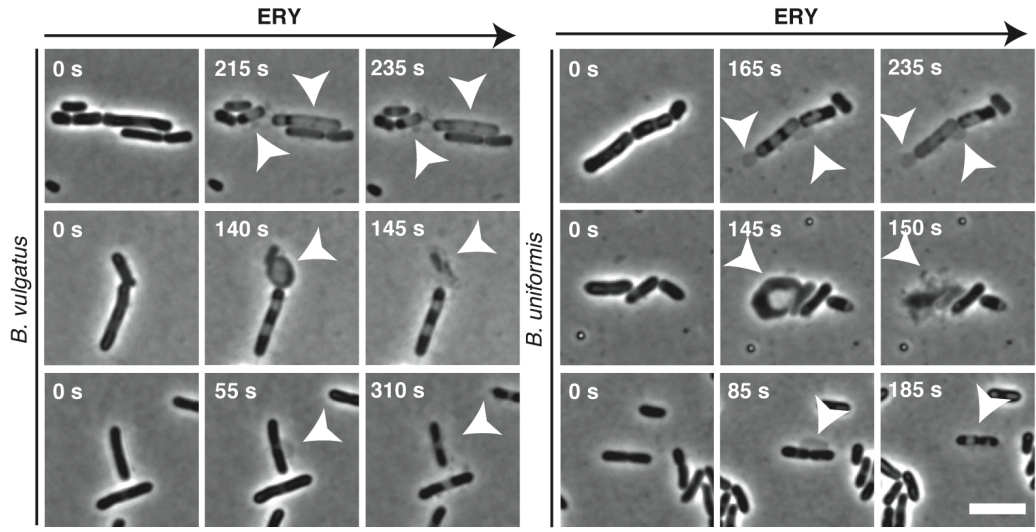

b

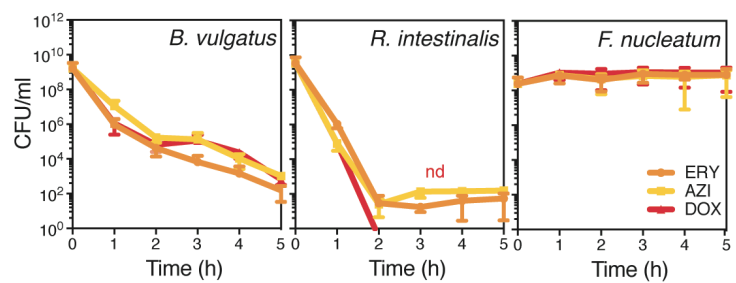

C
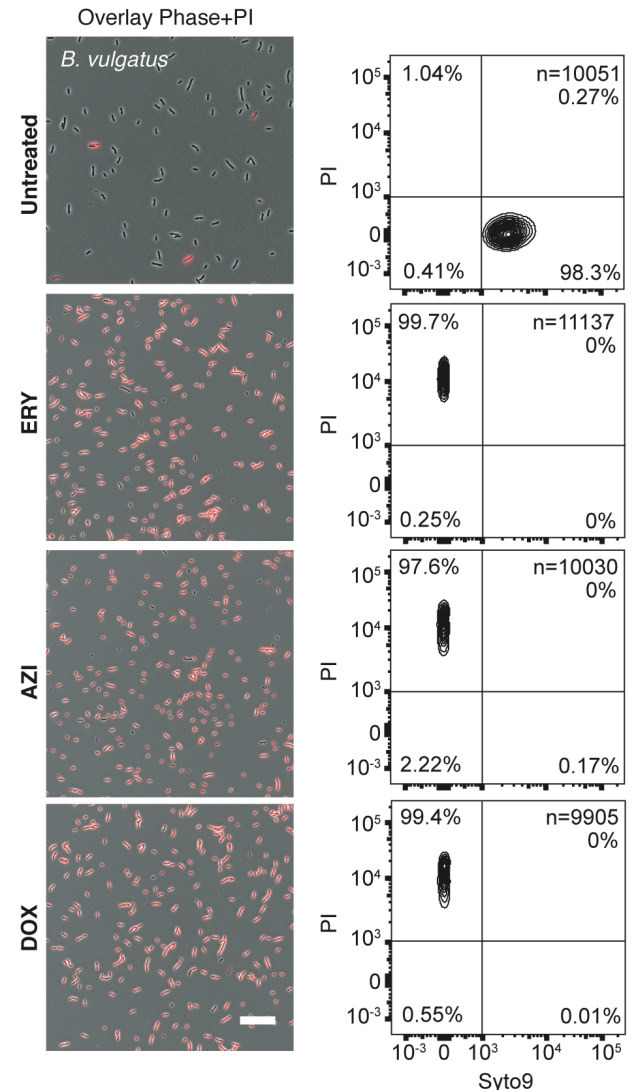

Figure 2

Figure 2 - Macrolides and tetracyclines kill human gut commensal species

a. The survival of 12 abundant gut microbe species was measured after a 5-hour treatment with a 5-fold MIC of erythromycin (ERY), azithromycin (AZI) or doxycycline (DOX). The survival was assessed by counting CFUs/ml before and after antibiotic treatment. The number of CFUs $/ \mathrm{ml}$ before treatment was set as $100 \%$. The detection limit for each experiment (gray bar) and the bactericidal threshold (shaded area) are indicated. Species are plotted according to phylogeny (IQTree, Methods) and in bold are noted the species that are used in later panels. The graph shows the mean+SD of 3 independent experiments. b. Time-kill curves of $B$. vulgatus, $R$. intestinalis and $F$. nucleatum after antibiotic treatments. Survival was assessed by CFU counting over a 5 hour-treatment of ERY, AZI or DOX. This graph shows the mean \pm SD of 3 independent experiments. Nd: non-detectable. Time-kill curves for the other tested gut microbes can be found in Extended Data Fig. 5. c. Live/dead staining of macrolide or tetracycline-treated B. vulgatus. The left panel shows an overlay of phase contrast and fluorescence microscopy images of propidium iodide (PI)-stained B. vulgatus before and 5 hours after ERY, AZI or DOX treatment. Cultures were concentrated before imaging; the scale bar is $10 \mu \mathrm{m}$. The right panel shows the corresponding quantification of live/dead-stained cells by flow cytometry with Syto9 on the x-axis (live cells) and PI on the $y$-axis (dead cells). Both the total number of measured events ( $\mathrm{n}$ ) and the percentage of cells found in each quadrant are indicated. d. Erythromycin induces lysis of $B$. vulgatus and $B$. uniformis. $B$. vulgatus and $B$. uniformis were grown for 3 hours before addition (yellow) or not (black) of $15 \mu \mathrm{g} / \mathrm{ml} \mathrm{ERY} \mathrm{treatment} \mathrm{(5-fold} \mathrm{MIC;} \mathrm{yellow)} \mathrm{as} \mathrm{indicated} \mathrm{by} \mathrm{the}$ arrow. Growth curves were acquired for 20 hours. This graph shows the mean $\pm S D$ (dotted line) of 3 independent experiments. e. Erythromycin induces blebbing, cytoplasmic shrinkage and lysis in $B$. vulgatus and $B$. uniformis. Phase contrast movies of $B$. vulgatus and $B$. uniformis were acquired after ERY treatment (5-fold MIC). Here shown 3 frames of 3 images per strain (time indicated in the upper left corner; $t=0$ when drug added). White arrows indicate blebs, cytoplasmic shrinkage and bacterial lysis; the scale bar is $5 \mu \mathrm{m}$. Movies are available in Supplementary Material (Movies 1-4). 
bioRxiv preprint doi: https://doi.org/10.1101/2020.01.09.893560; this version posted January 9, 2020. The copyright holder for this preprint (which was not certified by peer review) is the author/funder, who has granted bioRxiv a license to display the preprint in perpetuity. It is

a

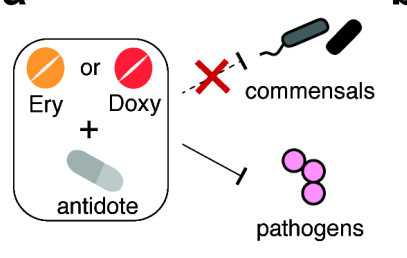

b

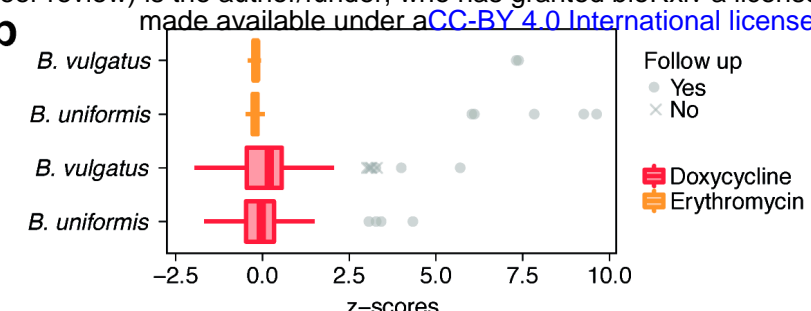

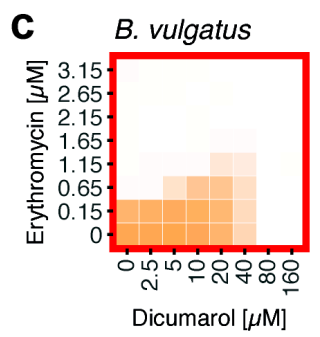

B. vulgatus

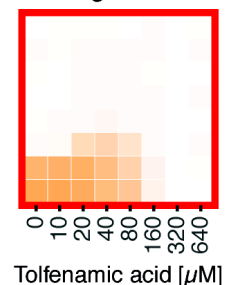

B. vulgatus

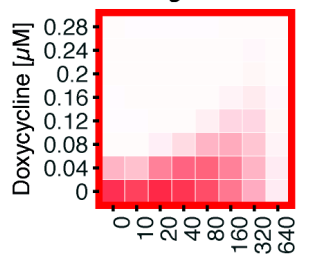

Diflunisal $[\mu \mathrm{M}]$

\section{d}
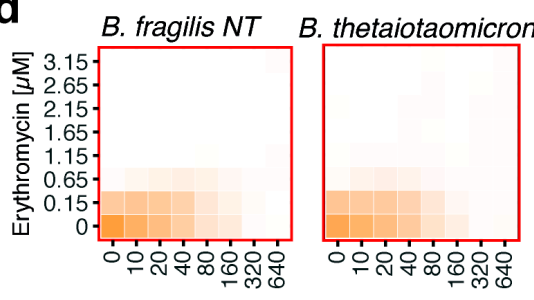

B. vulgatus

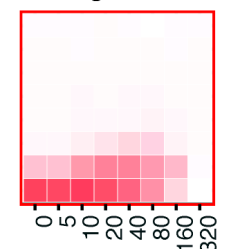

Iopanoic acid $[\mu \mathrm{M}]$

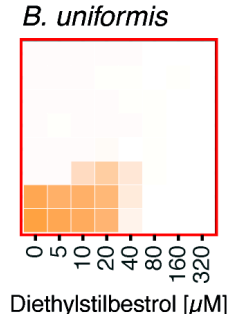

$B$. vulgatus

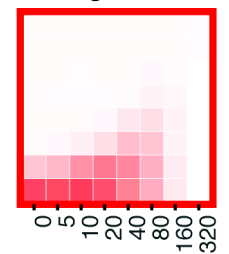

Tolfenamic acid $[\mu \mathrm{M}]$

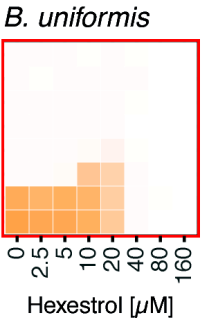

B. uniformis

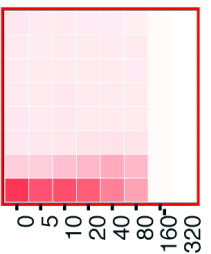

Tiratricol $[\mu \mathrm{M}]$

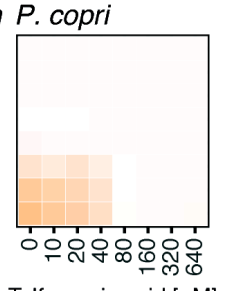

e
B. uniformis

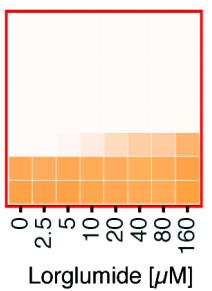

Tolfenamic acid $[\mu \mathrm{M}]$ Tolfenamic acid $[\mu \mathrm{M}]$ Tolfenamic acid $[\mu \mathrm{M}]$

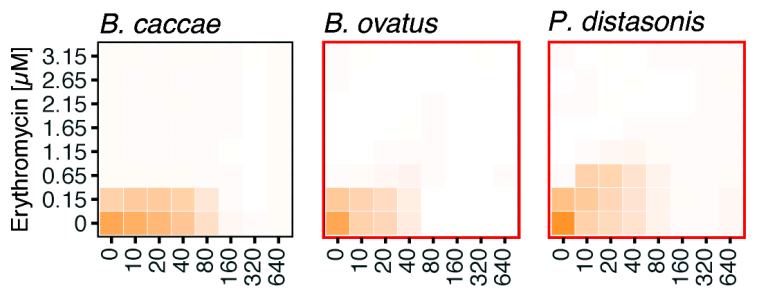

Tolfenamic acid $[\mu \mathrm{M}]$ Tolfenamic acid $[\mu \mathrm{M}]$ Tolfenamic acid $[\mu \mathrm{M}]$

S. aureus DSM20231

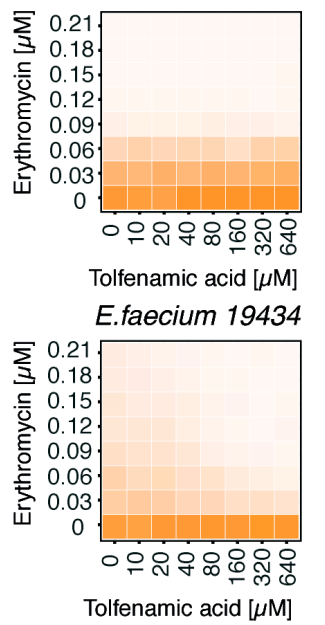

f
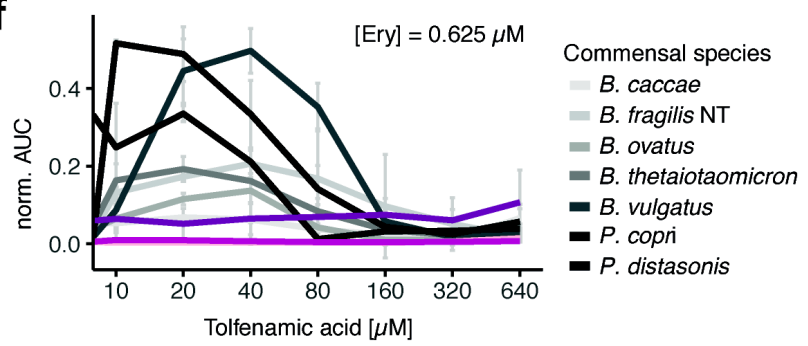

Pathogenic species

E. faecium ATCC 19434 [1.25 $\mu \mathrm{M}]$

- S. aureus DSM20231 [0.625 $\mu \mathrm{M}]$

- S. pneumoniae D39 [0.2 $\mu \mathrm{M}]$

\begin{tabular}{cc} 
AUC & AUC \\
\hline 1.00 & -1.00 \\
0.75 & 0.75 \\
0.50 & 0.50 \\
0.25 & 0.25 \\
0.00 & 0.00
\end{tabular}


bioRxiv preprint doi: https://doi.org/10.1101/2020.01.09.893560; this version posted January 9, 2020. The copyright holder for this preprint

(which was not certified by peer review) is the author/funder, who has granted bioRxiv a license to display the preprint in perpetuity. It is made available under aCC-BY 4.0 International license.

Tetracyclines

Oxazolidinone

Macrolides,

lincosamides and

streptogramins

Beta-lactams

Glycopeptides and lipoglycopeptides

(Fluoro-)quinolones

Miscellaneous agents

Aminoglycosides

Sulfonamides
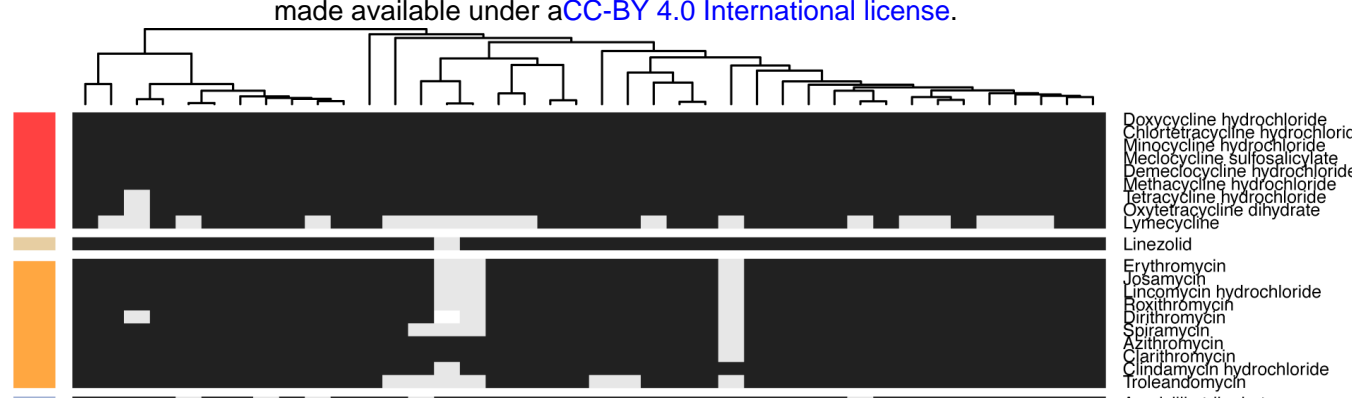

Cirithromycin
Troleamycin ndydrochloride
Tromycin
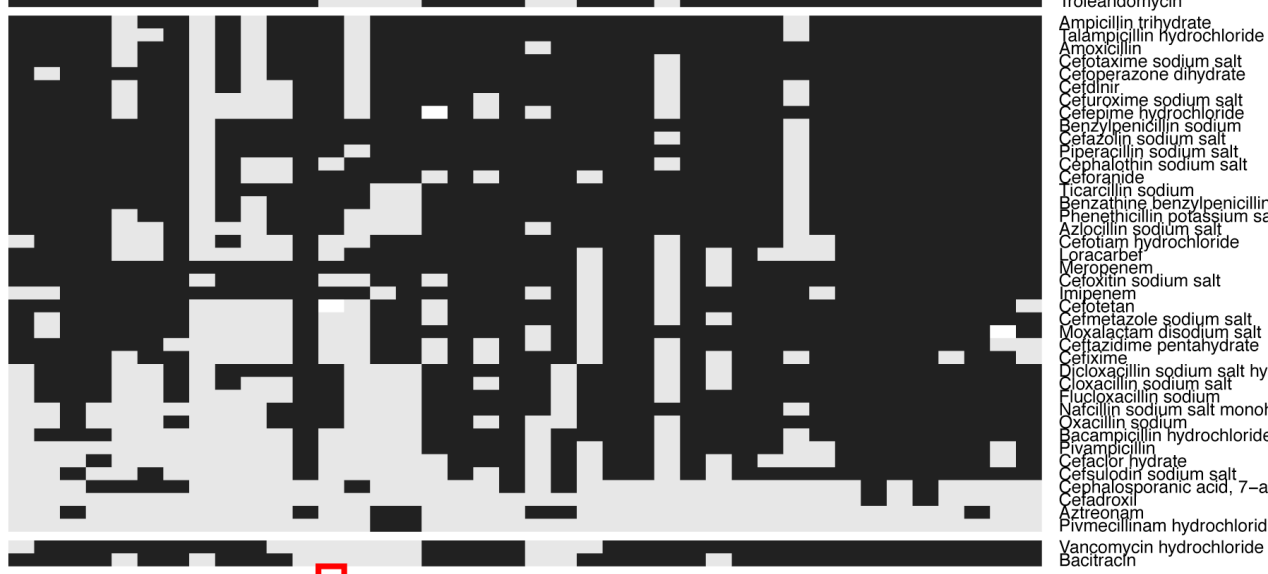

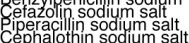

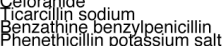

Azlocillin sodium salt
Céotian yodrochloride

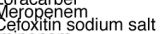

mipenem
ceotetean

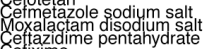

glcoxacilin sodium salt hydrate

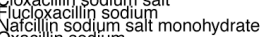

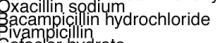

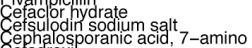

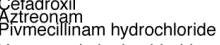

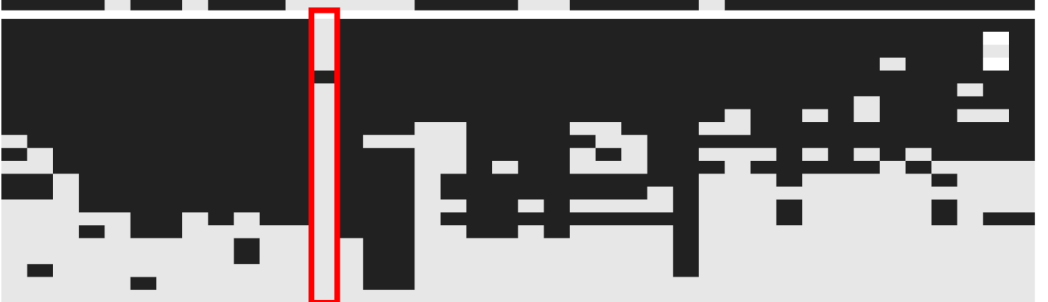

actn hy rochlonte

Cjprofloxacin hydrochloride monohydrate

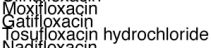

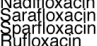

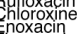

comefluine macin hydrochloride

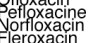

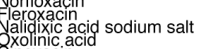

Cinoxacin
sipemilic acid
Silic acid

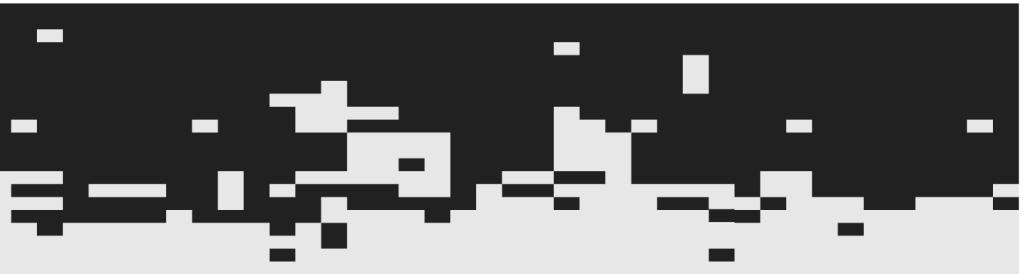

Chlloramphenicol

Fisapentine

finuroxazide
usidic accid sodium salt

Ornidaazole
Metrond

Tinidazole
clofazine
Colistinin sulfate

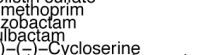

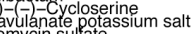

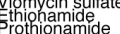
rapsonenamide razinamide

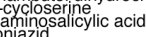

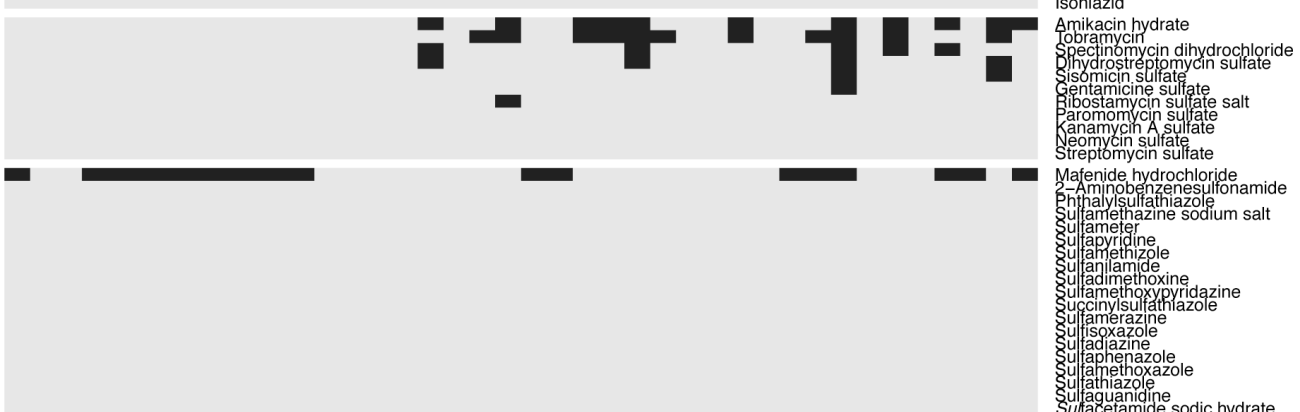

Amikacin hydrate

\%

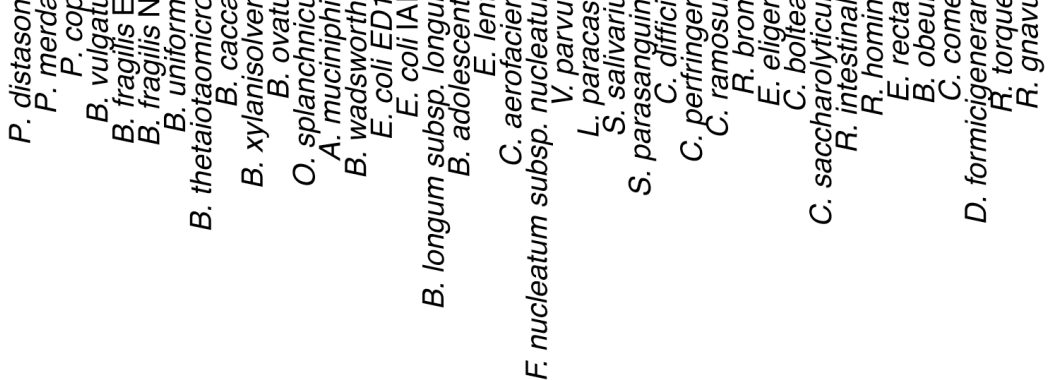

\section{Abx Sensitivity \\ sensitive \\ resistant}

\section{ED Figure 1}

Extended Data Figure 1 - Effects of 144 antibiotics on $\mathbf{4 0}$ human gut commensals

Heat map according to sensitivity or resistance of each strain to the respective antibiotic at a concentration of $20 \mu \mathrm{M}$. Antibiotics are grouped according to drug classes and species are clustered according to their responses across the 144 antibiotics tested. Data is replotted from ${ }^{4}$. Akkermansia muciniphila (Muc, DSM22959, type strain) is resistant to nearly all quinolone antibiotics (red box). We consolidated this finding by MIC determination for Ciprofloxacin (>32 $\mu \mathrm{g} / \mathrm{ml})$, Gatifloxacin $(>32 \mu \mathrm{g} / \mathrm{ml})$, Moxifloxacin $(>32 \mu \mathrm{g} / \mathrm{ml})$, Norfloxacin $(>256 \mu \mathrm{g} / \mathrm{ml})$ and Ofloxacin $(>32 \mu \mathrm{g} / \mathrm{ml})$. 
bioRxiv preprint doi: https://doi.org/10.1101/2020.01.09.893560; this version posted January 9, 2020. The copyright holder for this preprint

(which was not certified by peer review) is the author/funder, who has granted bioRxiv a license to display the preprint in perpetuity. It is made available under aCC-BY 4.0 International license.

Tetracyclines

Oxazolidinone

Macrolides,

lincosamides and streptogramins

Beta-lactams

Glycopeptides and lipoglycopeptides
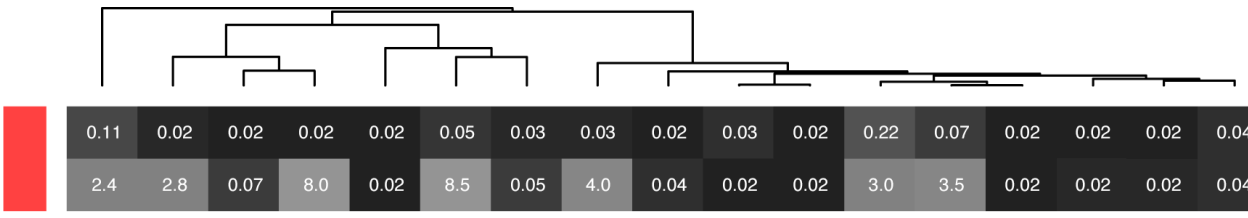

.

(1)

(Fluoro-)quinolones

Miscellaneous agents

\section{Aminoglycosides}

Sulfonamides
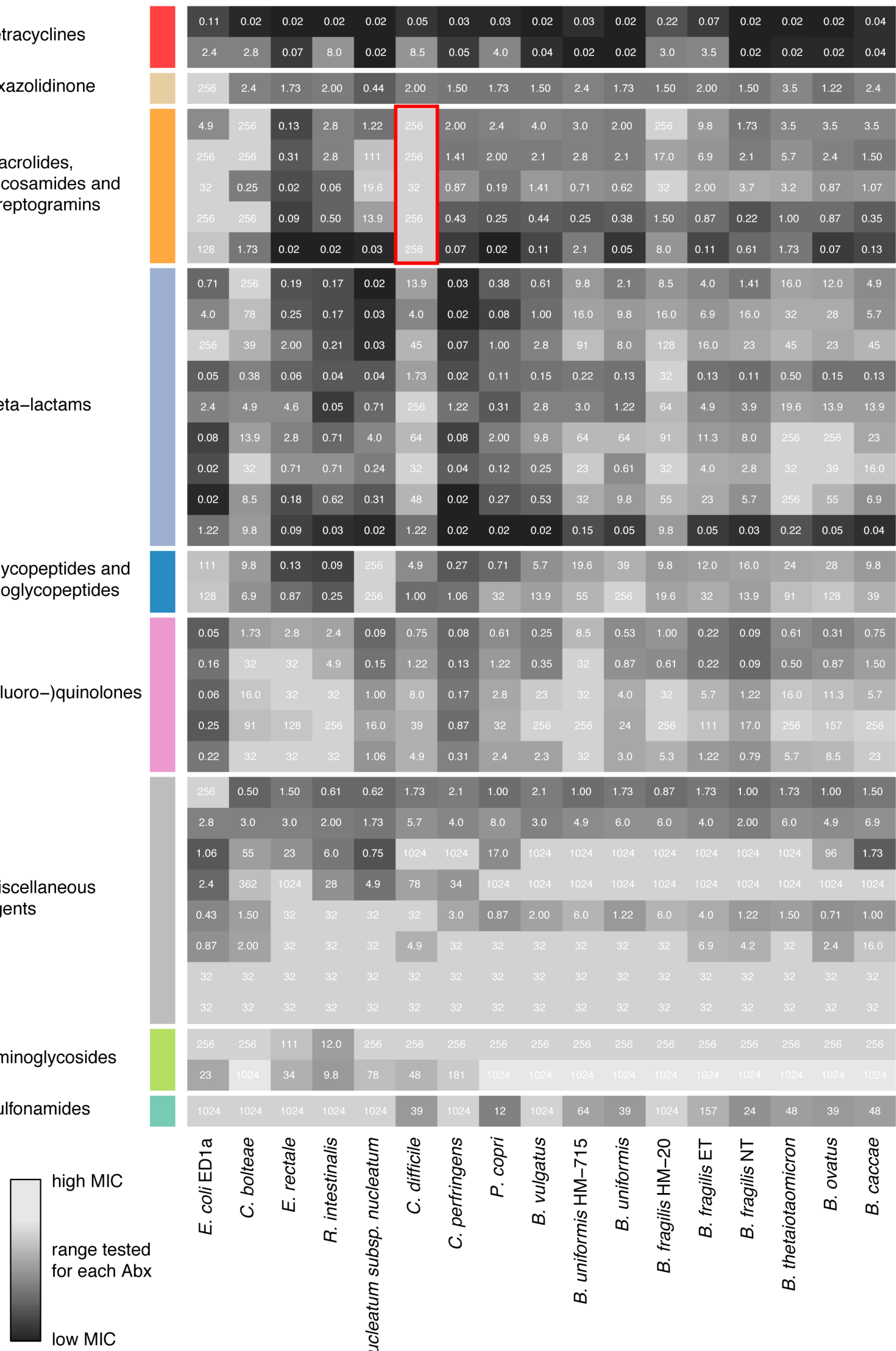

Tigecycline

$$
\text { . }
$$$$
\begin{array}{|l|l|l|l|l|}
\hline 2.4 & 1.73 & 2.00 & 0.44 & 2.00 \\
\hline 256 & 0.13 & 2.8 & 1.22 & \\
\hline 256 & 0.31 & 2.8 & 111 \\
\hline 0.25 & 0.02 & 0.06 & 19.6 \\
\hline 256 & 0.09 & 0.50 & 13.9 \\
\hline 1.73 & 0.02 & 0.02 & 0.03 \\
\hline
\end{array}
$$$$
\begin{array}{|lllllllllllll}
2.00 & 1.50 & 1.73 & 1.50 & 2.4 & 1.73 & 1.50 & 2.00 & 1.50 & 3.5 & 1.22 & 2.4 \\
\hline
\end{array}
$$

Doxycycline

Linezolid
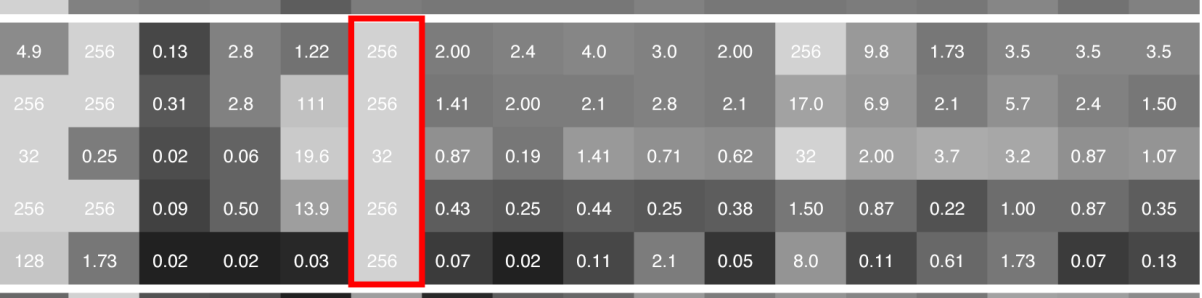

Azithromycin

Erythromycin

Spiramycin

Clarithromycin

Clindamycin

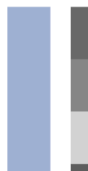
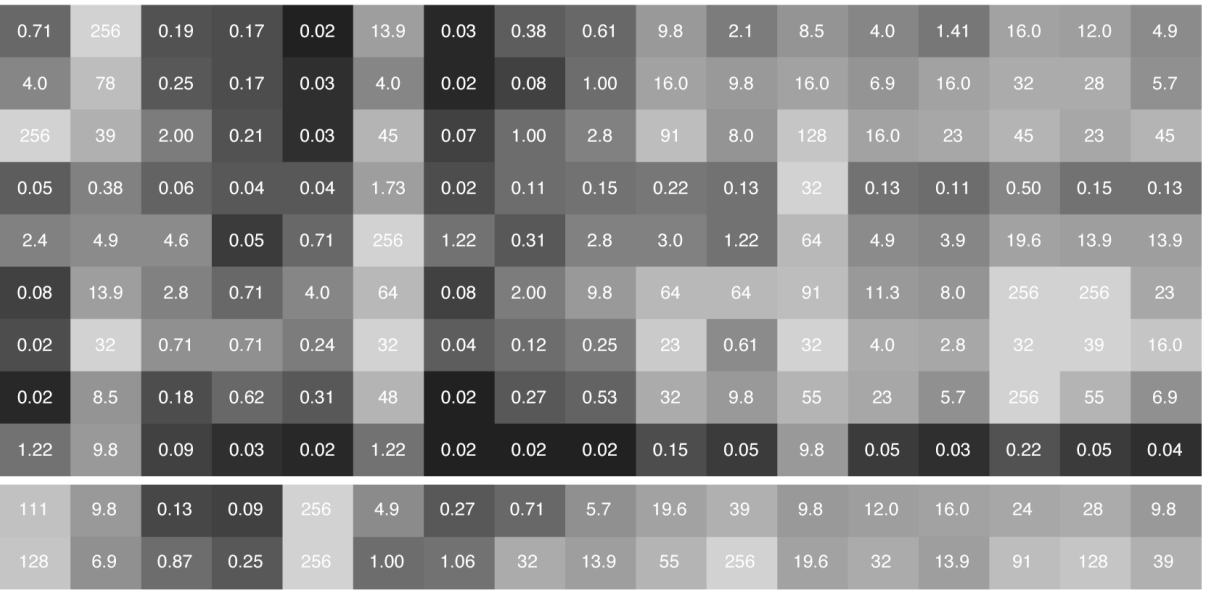

Piperacillin

Amoxicillin

Oxacillin

Meropenem

Cefoxitin

Ceftazidime

Cefotaxime

Ceftriaxone

Amoxicillin +

Clavulanic Acid

Teicoplanin

Vancomycin
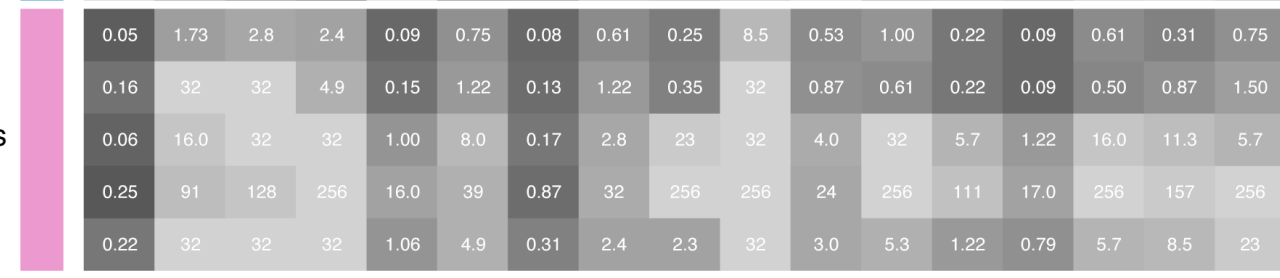

Gatifloxacin

Moxifloxacin

Ciprofloxacin

Norfloxacin

Ofloxacin

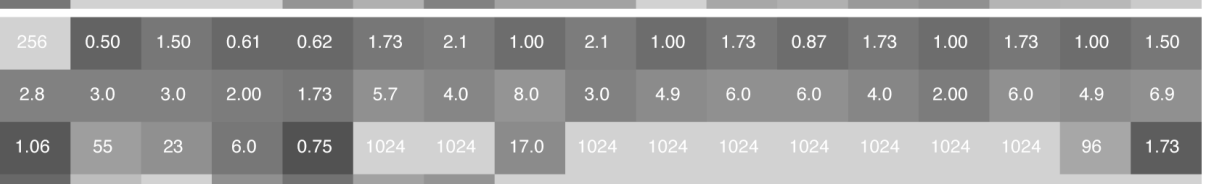

Metronidazole

Chloramphenicol

Colistin

Fosfomycin

Trimethoprim +

Sulfamethoxazole

Trimethoprim

Itraconazole

Ketoconazole

Amikacin

Tobramycin

Sulfamethoxazole

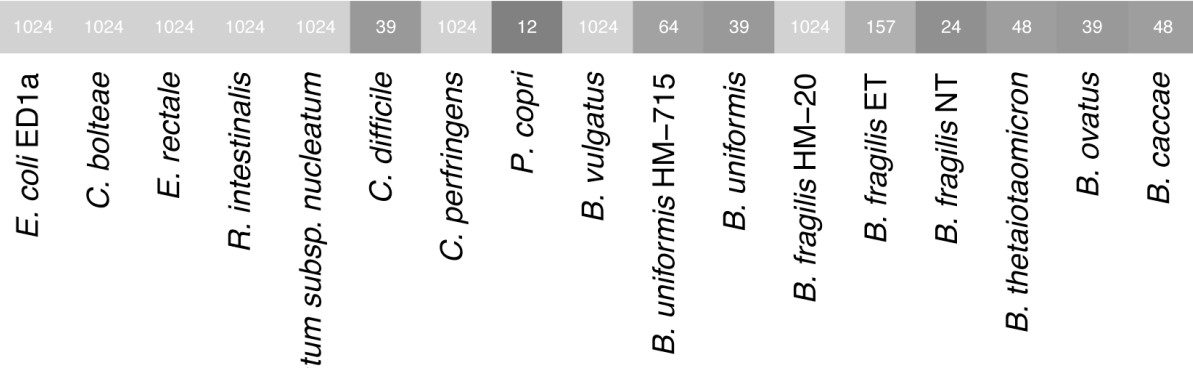

ED Figure 2

Extended Data Figure 2 - MICs for 17 species on $\mathbf{3 5}$ antimicrobials

Heat map depicts MICs for each drug-strain pair in $\mu \mathrm{g} / \mathrm{ml}$. Heat map color gradient is adjusted to the MICs concentration range tested on the respective MIC test strip. Black depicts sensitivity and light grey indicates resistance. Mean values across two biological replicates are shown (Suppl. Table 3). C. difficile is particularly resistant to all tested macrolides and clindamycin (red box). 
bioRxiv preprint doi: https://doi.org/10.1101/2020.01.09.893560; this version posted January 9, 2020. The copyright holder for this preprint (which was not certified by peer review) is the author/funder, who has granted bioRxiv a license to display the preprint in perpetuity. It is
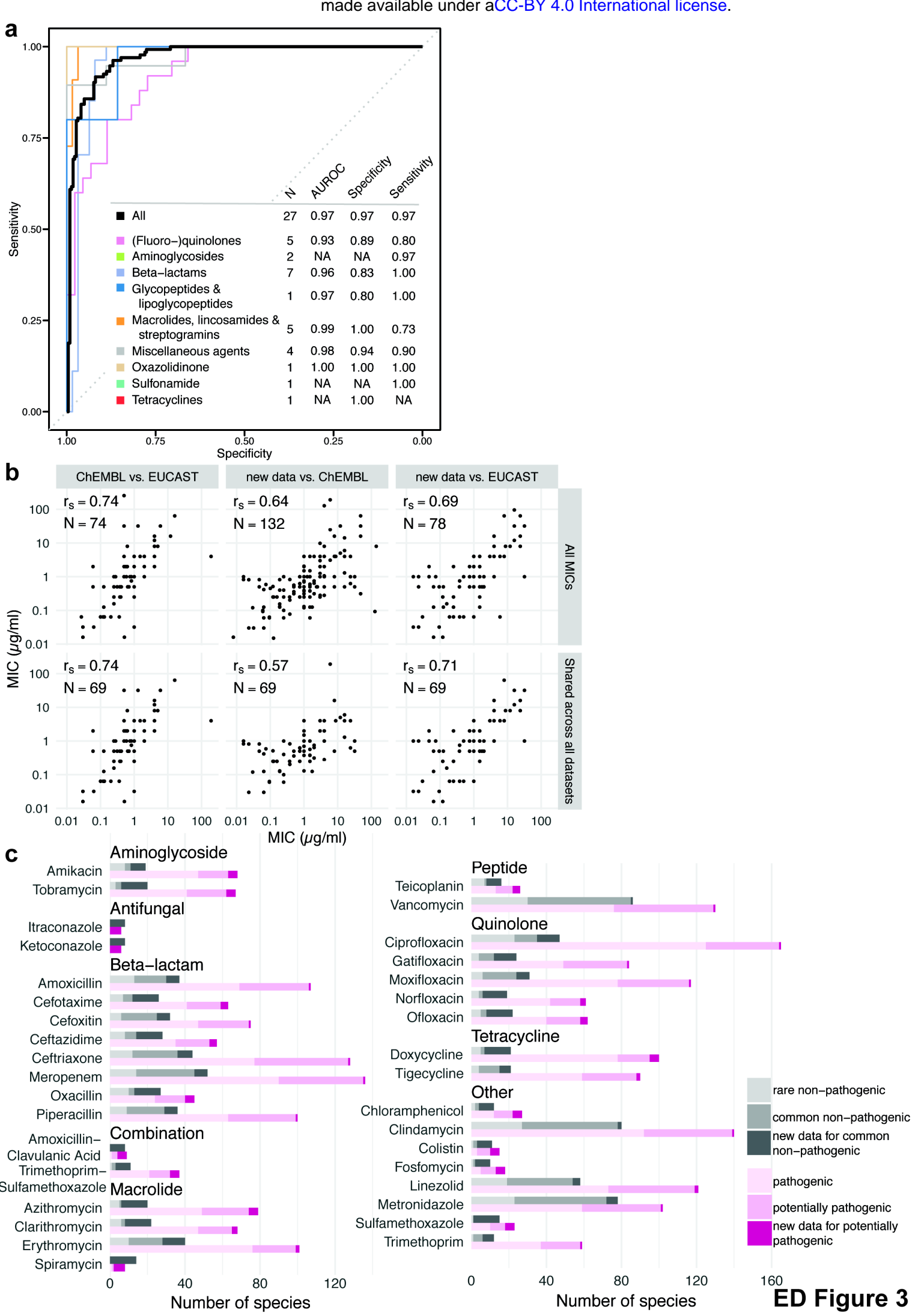

Extended Data Figure 3 - MIC dataset validates antibiotic sensitivity profiles from the screen dataset and is consistent with publically available MICs.

a. Receiver operating characteristic (ROC) curve analysis was performed to evaluate sensitivity and specificity of the screen ${ }^{4}$ using the MIC dataset. Results from the screen were considered as validated if MICs were below/above the $20 \mu \mathrm{M}$ antibiotic concentration that was tested in the screen (allowing a two-fold error margin). $\mathrm{N}$ is the number of antibiotics that we tested both in the screen and determined MICs for, AUROC is the area under the characteristic ROC. TN denotes true negatives, FP false positives, TP true positives, FN false negatives.

b. Comparison including Spearman correlation coefficients of the MICs from this study to MICs from the ChEMBL ${ }^{27}$ and EUCAST ${ }^{24}$ databases. Panels in the upper row: comparison between all MICs that are shared between the two indicated datasets. Panels in the lower row: comparison of the $69 \mathrm{MICs}$ that are shared across all three datasets. Despite experimental differences, our MICs correlate well with available EUCAST/ ChEMBL data.

c. Number of the sum of new (this study) and already available MICs (EUCAST/ ChEMBL) per drug according to antibiotic class and prevalence/virulence of the bacterial species. The new dataset expands MICs across the board and specifically fills the knowledge gap on nonpathogenic species. 
bioRxiv preprint doi: https://doi.org/10.1101/2020.01.09.893560; this version posted January 9, 2020. The copyright holder for this preprint (which was not certified by peer review) is the author/funder, who has granted bioRxiv a license to display the preprint in perpetuity. It is

a Screen

4 beta-lactams

$\times 7$ Bacteroides spp.

(8 strains)

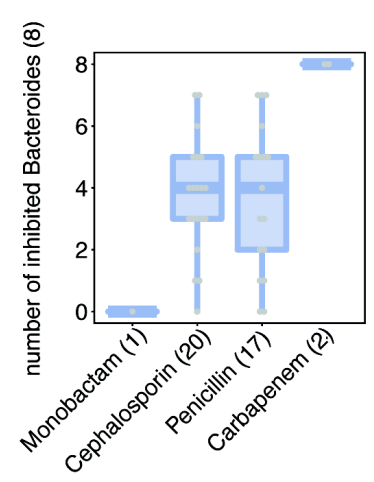

b

\section{MICs}

8 beta-lactams $x$ 12 Bacteroides spp. (19 strains)

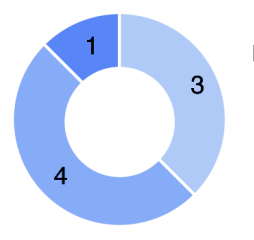

beta-lactam subclasses Carbapenems

Cephalosporins Penicillins

d
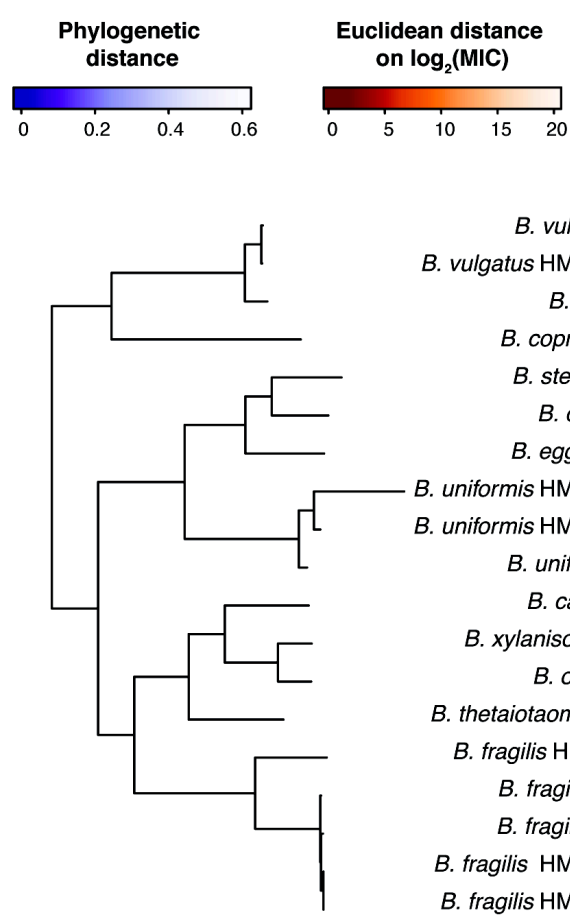

\footnotetext{
B. vulgatus

B. vulgatus $\mathrm{HM}-720$

B. dorei

B. coprocola

B. stercoris

B. clarus

B. eggerthii

B. uniformis $\mathrm{HM}-716$

B. uniformis $\mathrm{HM}-715$

B. uniformis

B. caccae

B. xylanisolvens

B. ovatus

B. thetaiotaomicron

B. fragilis $\mathrm{HM}-20$

B. fragilis $\mathrm{ET}$

B. fragilis NT

B. fragilis $\mathrm{HM}-710$

B. fragilis $\mathrm{HM}-709$
}

B. fragilis ET

B. clarus

B. fragilis NT

B. caccae

B. uniformis

B. coprocola

B. vulgatus

B. eggerthii

B. vulgatus $\mathrm{HM}-720$

B. ovatus

B. xylanisolvens

B. thetaiotaomicron

B. fragilis $\mathrm{HM}-709$

B. fragilis $\mathrm{HM}-710$

B. uniformis $\mathrm{HM}-715$

B. uniformis $\mathrm{HM}-716$

B. dorei

B. stercoris

B. fragilis $\mathrm{HM}-20$

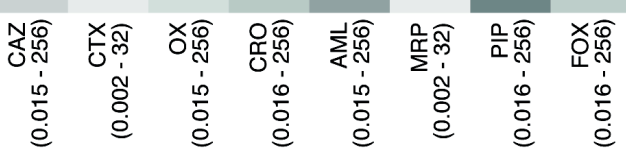



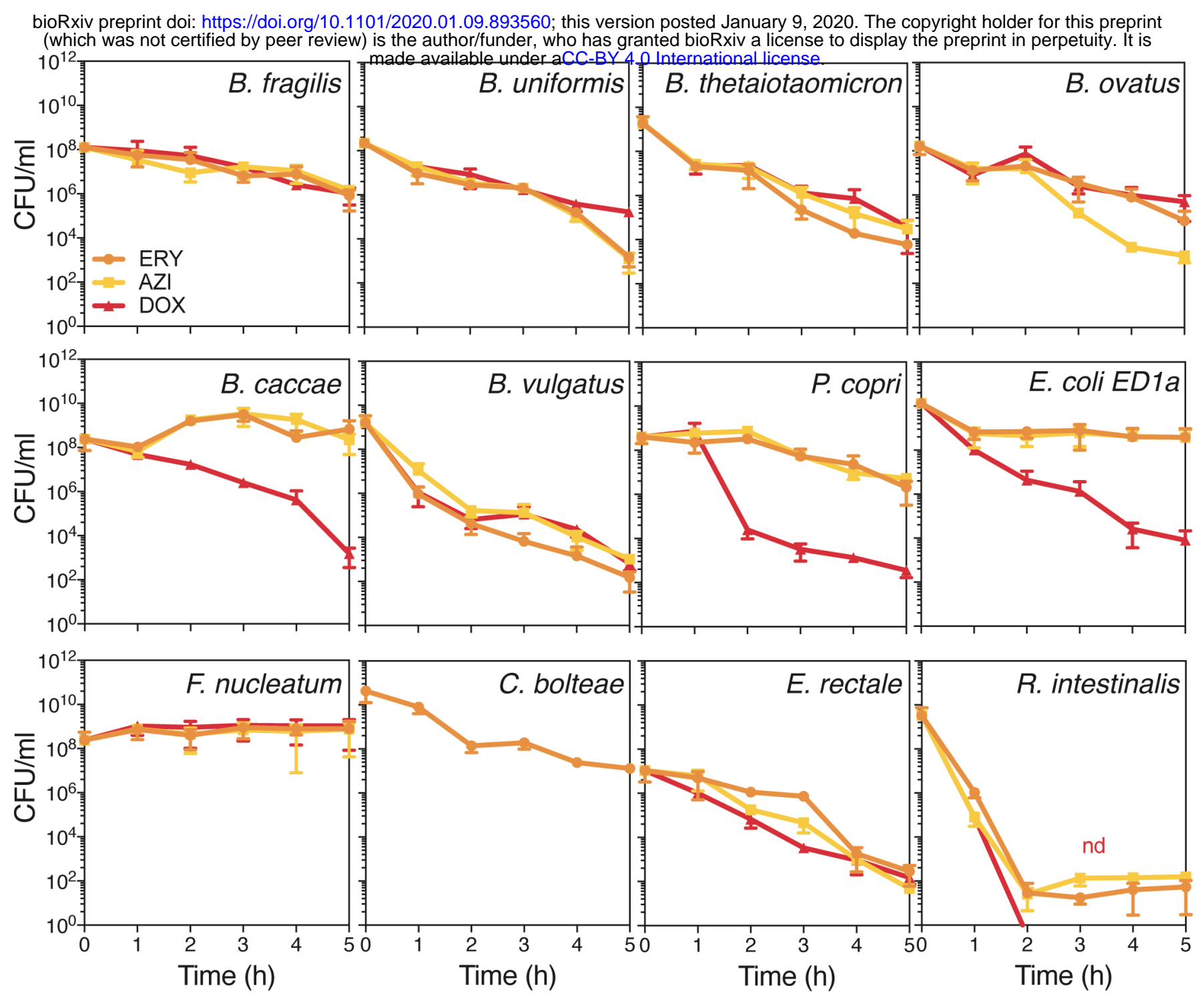

ED Figure 5

Extended Data Figure 5 - Time-kill curves of 12 abundant gut microbes after treatment with macrolides and tetracyclines.

Survival of 12 abundant gut microbes was assessed by CFU counting over a 5 hour-treatment of either ERY, AZI or DOX. This graph shows the mean $\pm S D$ of 3 independent experiments. 
bioRxiv preprint doi: https://doi.org/10.1101/2020.01.09.893560; this version posted January 9, 2020. The copyright holder for this preprint

(which was not certified by peer review) is the author/funder, who has granted bioRxiv a license to display the preprint in perpetuity. It is made available under aCC-BY 4.0 International license.

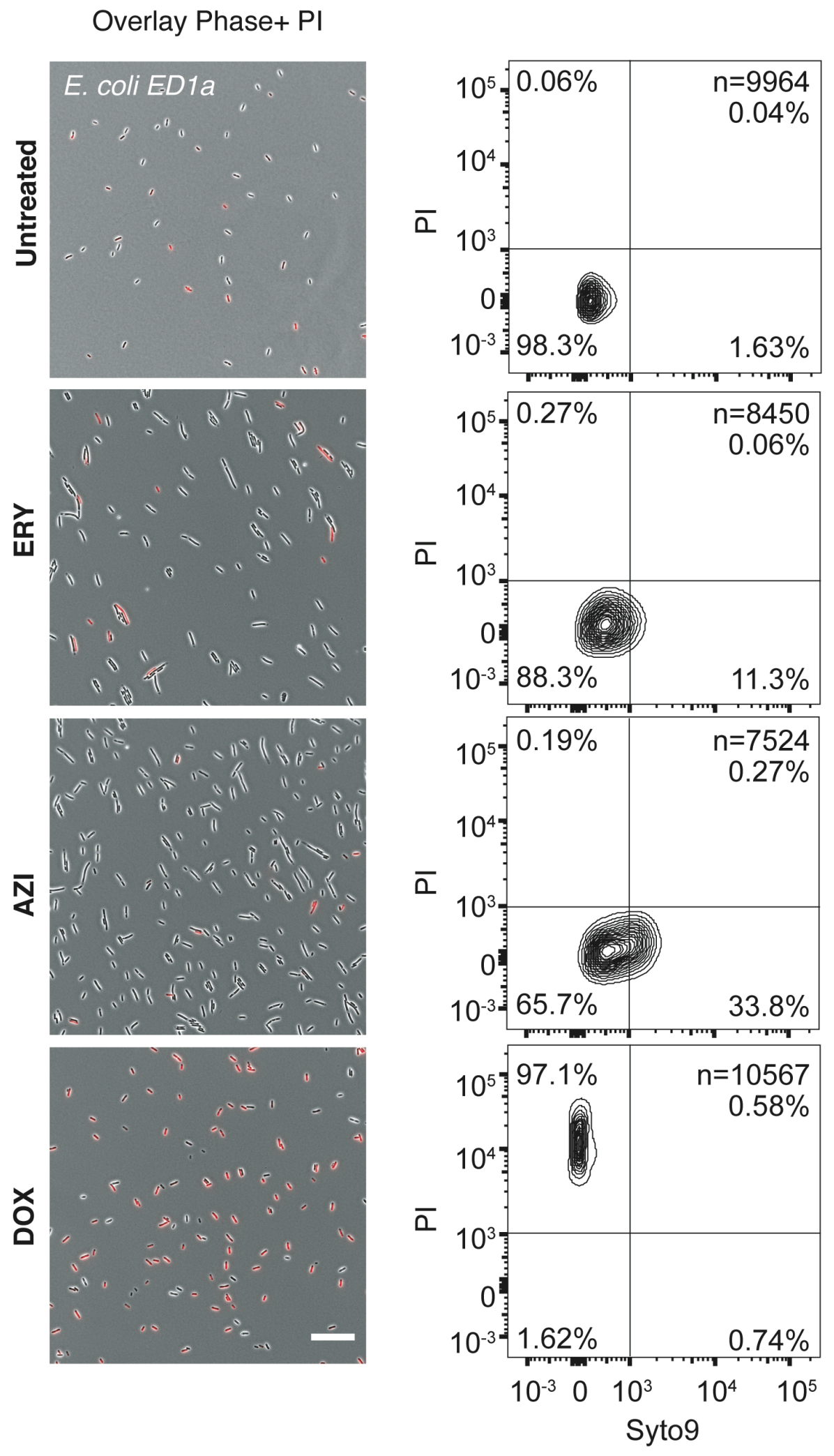

\section{ED Figure 6}

Extended Data Figure 6 - Live/dead staining of macrolide or tetracycline-treated E. coli ED1a.

The left panel shows an overlay of phase contrast and fluorescence microscopy images of propidium iodide (PI)-stained E. coli ED1a before and 5 hours after ERY, AZI or DOX treatments. The number of cells on each frame has no meaning, as cultures were concentrated before imaging; the scale bar is $10 \mu \mathrm{M}$. The right panel shows the corresponding quantification of live/dead-stained cells by flow cytometry with Syto 9 on the $\mathrm{x}$ axis (live cells) and PI on the $y$-axis (dead cells). Both the total number of measured events $(n)$ and the percentage of cells found in each quadrant are indicated on the graphs. 
bioRxiv preprint doi: https://doi.org/10.1101/2020.01.09.893560; this version posted January 9, 2020. The copyright holder for this preprint (which was not certified by peer review) is the author/funder, who has granted bioRxiv a license to display the preprint in perpetuity. It is

2 b made available under aCC-BY 4.0 International license.

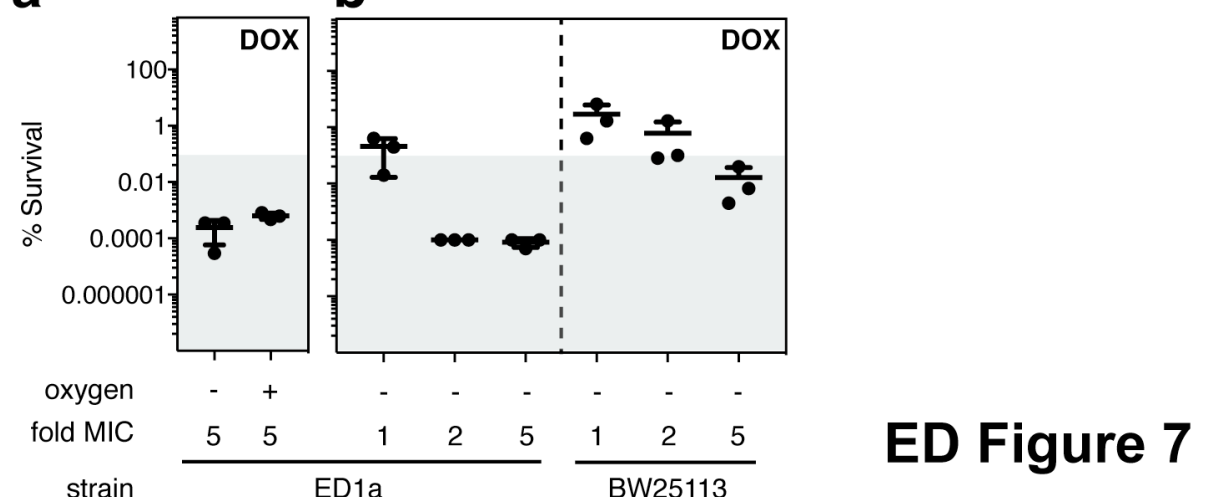

Extended Data Figure 7 - Effect of oxygen and strain specificity on survival after doxycycline treatment

a. The survival of $E$. coli ED1a was assessed after a 5-hour treatment with 5-fold MIC of DOX in the presence or absence of oxygen. Killing was similarly effective in both conditions.

b. The survival of $E$. coli ED1a and E. coli BW25113 were assessed after a 5-hour treatment with 1, 2 and 5-fold MIC of DOX in MGAM medium in anaerobic conditions. The lab strain is more resistant to killing with doxycycline becoming boarder-line bactericidal at higher MICs. 
bioRxiv preprint doi: https://doi.org/10.1101/2020.01.09.893560; this version posted January 9, 2020. The copyright holder for this preprint (which was not certified by peer review) is the author/funder, who has granted bioRxiv a license to display the preprint in perpetuity. It is
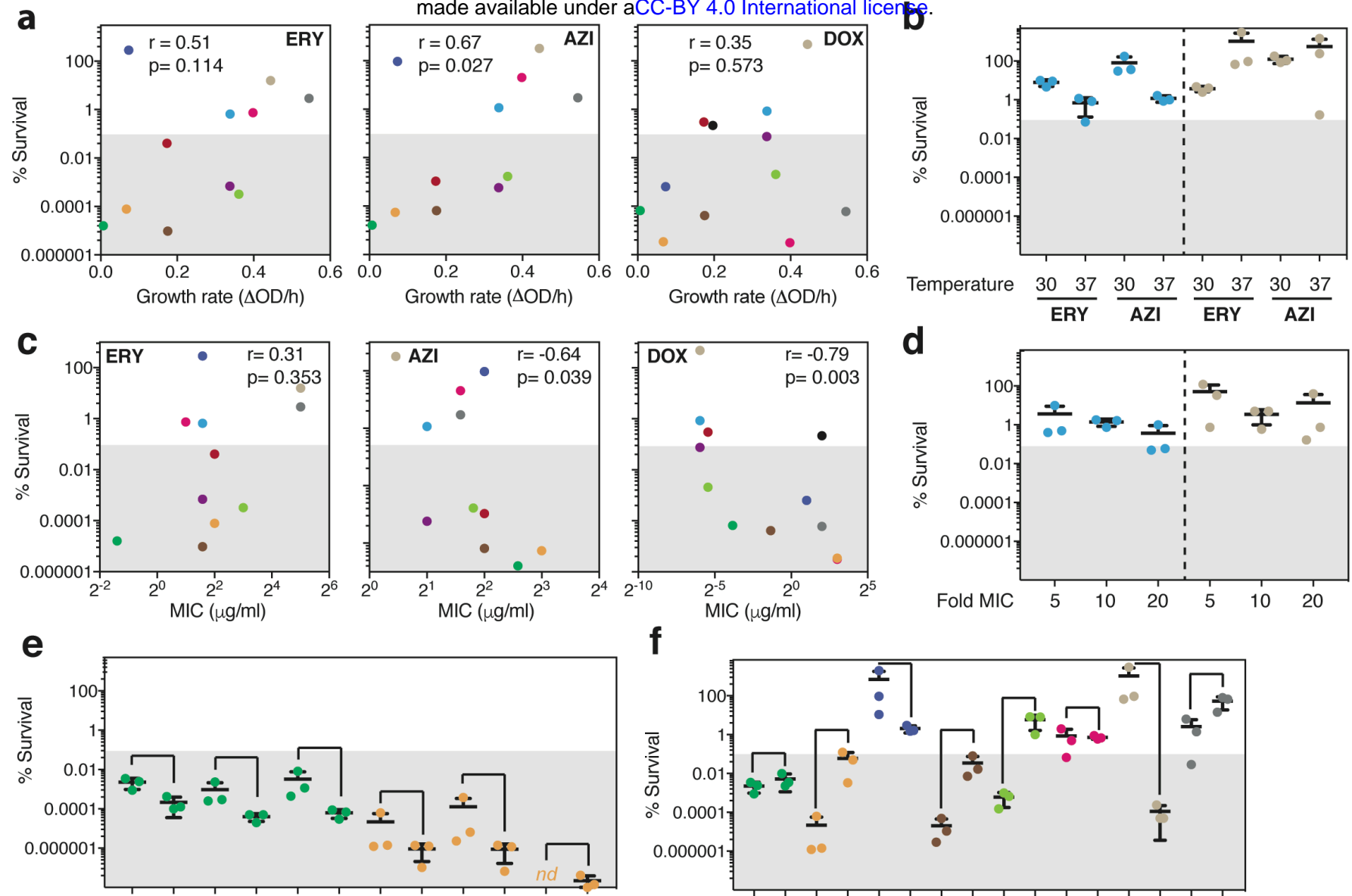

Growth $\frac{2 \mathrm{~h} 3 \mathrm{~h}}{\mathrm{ERY}} \frac{2 \mathrm{~h} 3 \mathrm{~h}}{\mathrm{AZI}} \frac{2 \mathrm{~h} 3 \mathrm{~h}}{\mathrm{DOX}} \frac{2 \mathrm{~h} 3 \mathrm{~h}}{\mathrm{ERY}} \frac{2 \mathrm{~h} 3 \mathrm{~h}}{\mathrm{AZI}} \frac{2 \mathrm{~h} 3 \mathrm{~h}}{\mathrm{DOX}}$

Phase $\begin{array}{llllllllllllllll} & E & S & E & S & E & S & E & S & E & S & E & S & E & S & E\end{array}$

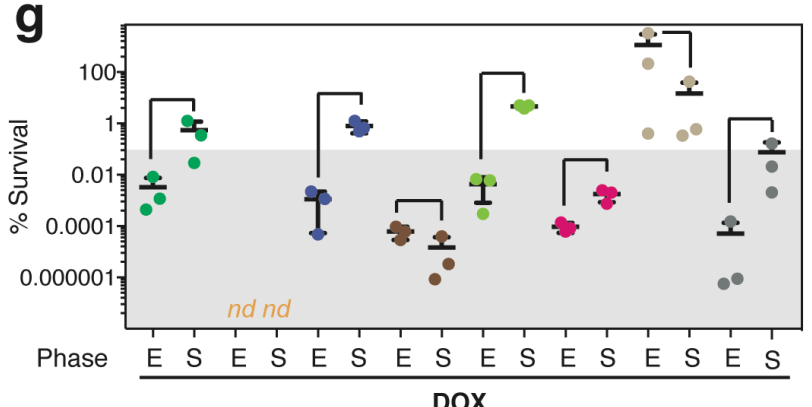

$\begin{array}{ll}\text { E. rectale } & \text { B. uniformis } \\ \text { R. intestinalis } & \text { B. fragilis } \\ \text { B. caccae } & \text { B. tethaiotaomicron } \\ \text { B. ovatus } & \text { P. copri } \\ \text { B. vulgatus } & \text { F. nucleatum } \\ \text { C. bolteae } & \text { E. coli ED1a }\end{array}$

ED Figure 8

Extended Data Figure 8 - Assessing potential confounding factors for the killing capacities of erythromycin, azithromycin and doxycycline

a. Scatter plot of individual bacterial growth rates and percentage survival after a 5-hour treatment with 5-fold MIC of ERY, AZI or DOX treatments. $r$ indicates the Spearman correlation coefficient. Tested species are color-coded here and in all panel thereafter as indicated in the bottom of this figure. Positive correlations for macrolides were tested further in $\mathbf{b}$ to check if changing growth rate in same species affects percentage killed.

b. The survival of $B$. fragilis (blue) and $F$. nucleatum (beige) were assessed after a 5-hour macrolide treatment (5-fold MIC of ERY and AZI) at either $30^{\circ} \mathrm{C}$ (slow growth) or $37^{\circ} \mathrm{C}$ (fast growth) to test the effect of slowing down growth on survival. No significant change observed. This graph shows the mean $\pm \mathrm{SD}$ of three independent experiments.

c. Scatter plot of MICs and percentage survival after a 5-hour treatment with 5 -fold MIC of ERY, AZI or DOX treatments. $r$ indicates the Spearman correlation coefficient. Doxycycline exhibited a strong and significant anti-correlation, that is that species which were more sensitive to doxycycline (lower MIC) were not killed when they were treated with 5-fold MIC concentrations. Thus, we tested further whether increasing the drug concentration in some of those sensitive strains decreased the \% of survival (panel d).

d. The survival of $B$. fragilis (blue) and $F$. nucleatum (beige) were assessed after a 5-hour treatment with increasing concentrations of DOX (5, 10 or 20- fold of MIC) to test whether higher concentrations of DOX induced more killing. This seemed not be the case. This graph shows the mean \pm SD of three independent experiments.

e. To evaluate whether outgrowth of stationary phase and homogeneity of population affected our results, we selected two slow-growing strains, $E$. rectale and $R$. intestinalis and grew for 2 or 3 hours after being diluted from an overnight culture to an of $O D_{578} 0.01$. Both strains were then treated for 5 hours with 5 -fold MIC of ERY, AZI or DOX and their survival was assessed to test the impact of the growth phase on the percentage survival. Although slight differences were observed and 3h grown cultures were killed more effectively (presumably because more cells had exited stationary phase and were growing exponentially by then), the general trends remained the same. If anything, this means that we are underestimating the killing for slow-growers, since we performed all other experiments with 2 hours outgrowth. This graph shows the mean $\pm S D$ of three independent experiments.

f. The survival of 8 selected gut microbes was measured after treating cells in exponential phase $(E-2$ hours after dilution from an overnight culture) or in stationary phase (S - overnight growth) with 5-fold MIC of ERY for 5 hours to test the impact of the growth phase on the percentage survival. As expected, survival is higher in stationary phase for half of the strains, but in some cases stationary phase cells were as or more sensitive than exponentially growing cells - this is the case for $B$. caccae and $F$. nucleatum. This graph shows the mean $\pm S D$ of three independent experiments.

g. Same as in $\mathbf{f}$ but with DOX. Similar effects observed as in $\mathrm{f}$, with more than half of strains becoming more resistant in stationary phase. 
bioRxiv preprint doi: https://doi.org/10.1101/2020.01.09.893560; this version posted January 9, 2020. The copyright holder for this preprint

(which was not certified by peer review) is the author/funder, who has granted bioRxiv a license to display the preprint in perpetuity. It is

a made available under aCC-BY 4.0 International license. Experimental setup Tested combinations Tested species

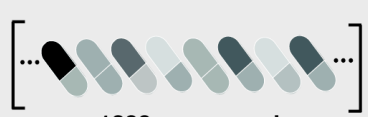

$\sim 1200$ compounds

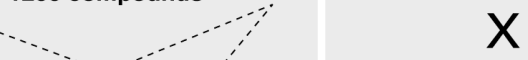

Erythromycin

ERY DOX

2 replicates

b

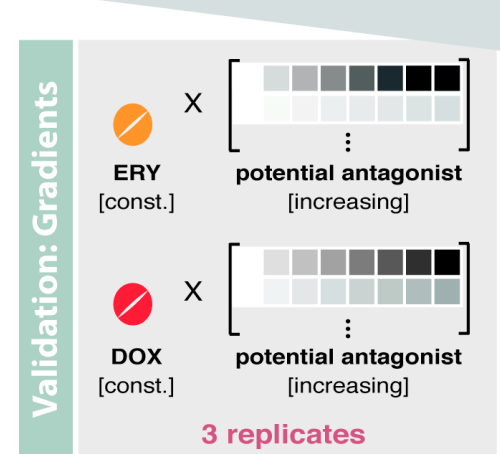

Prestwick library

$\begin{array}{ll}{[20 \mu \mathrm{M}]} & \text { B. vulgatus } \\ & \text { B. uniformis }\end{array}$

or

[just above MIC]

\section{Validate strongest hits}

14 combinations

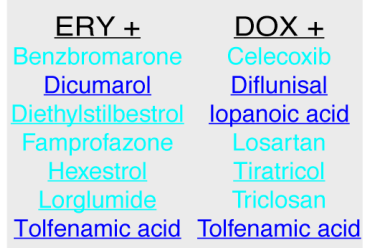

Selection criteria:

high abundance \&

prevalence in

the healthy gut
19 potential antagonists

Fig. 3b

Suppl. Table $\mathbf{S 4}$

C Checkerboard on 9 validated antagonisms

B. vulgatus

B. uniformis

Validated

9 antagonisms $(10-80 \mu \mathrm{M})$

ED Fig. 10a
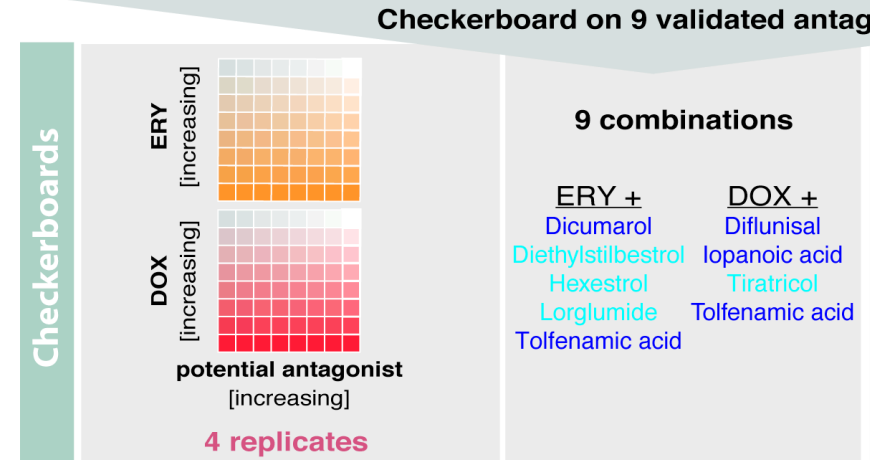

d

Expand species tested for $\mathbf{2}$ strongest antagonisms per antibtiotic

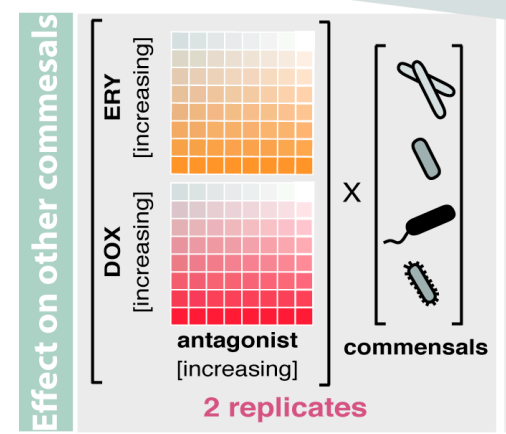

\section{4 combinations}

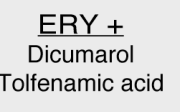

$\mathrm{DOX}+$

Diflunisal

Tolfenamic acid
B. vulgatus

B. uniformis

B. caccae

B. fragilis NT

B. ovatus

B. thetaiotaomicron

E. rectale

O. splanchnicus

P. copri

P. distasonis

e

Expand to pathogens for Ery combinations

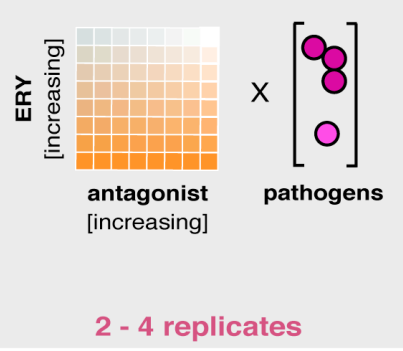

2 combinations

ERY +

Dicumarol

Tolfenamic acid
S. aureus

S. pneumoniae

E. faecium
Fig. 3c
Fig. 3e+f / ED Fig.12

Fig. 3d / ED Fig. 11

\section{ED Figure 9}

Extended Data Figure 9 - Schematic overview of screen for microbiome-protective antibiotic antagonisms Workflow with decision process on which antagonist to move on to next evaluation step. 
bioRxiv preprint doi: https://doi.org/10.1101/2020.01.09.893560; this version posted January 9,2020 . The copyright holder for this preprint (which was not certified by peer review) is the author/funder, who has granted bioRxiv a license to display the preprint in perpetuity. It is

a

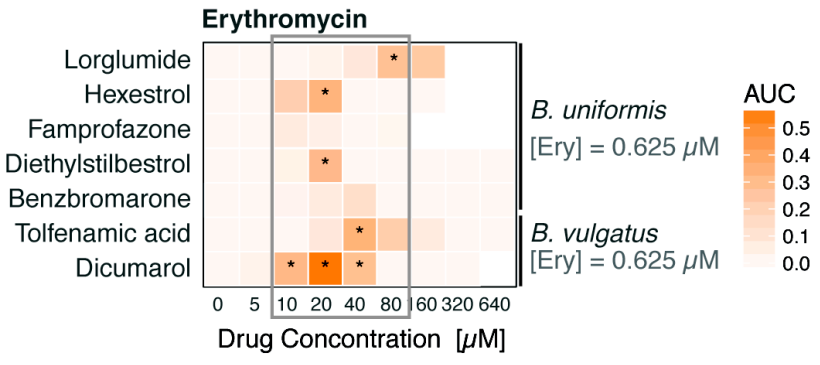

b

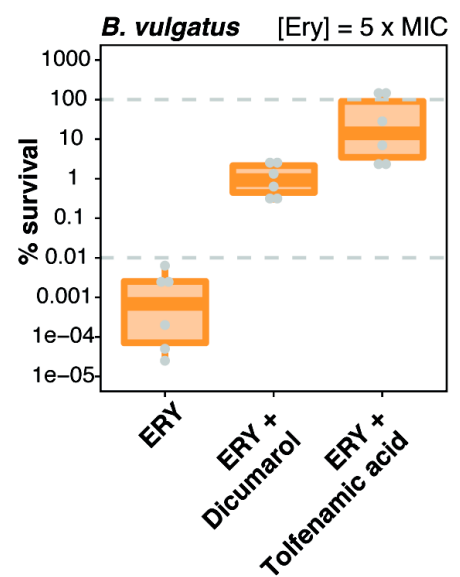

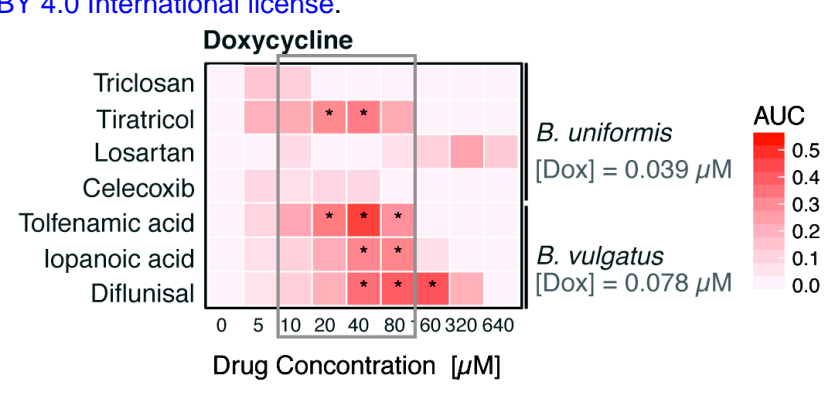

B. vulgatus $[\mathrm{Dox}]=5 \times \mathrm{MIC}$

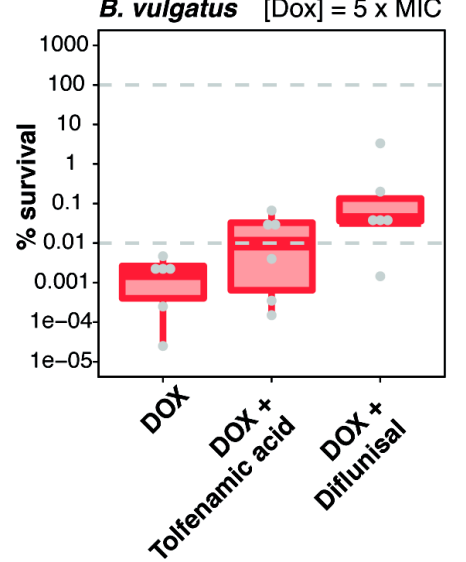

ED Figure 10

Extended Data Figure 10 - Validation of potential microbiome-protective antagonists

a. Validation of the strongest antagonists in independent experiments. Erythromycin and doxycycline concentrations were kept constant $([E R Y]=0.625 \mu \mathrm{M},[\mathrm{DOX}]=0.039 / 0.078 \mu \mathrm{M})$ and concentration ranges were tested for antagonist. Asterisks indicate that at least $25 \%$ of the bacterial growth (compared to no drug controls) could be rescued by the antagonist at a given concentration. Heat map depicts median AUCs across triplicates.

b. Percentage of surviving $B$. vulgatus cells were determined after $5 \mathrm{~h}$ incubation with either erythromycin $(3.25 \mu \mathrm{M})$ or doxycycline $(0.4 \mu \mathrm{M})$ alone or in presence of the antagonist dicumarol $(20 \mu \mathrm{M})$, tolfenamic acid $(40 \mu \mathrm{M})$ or diflunisal $(80 \mu \mathrm{M})$. Data is based on 3 independent experiments. Boxplots are plotted as in Figure 1d. 
bioRxiv preprint doi: https://doi.org/10.1101/2020.01.09.893560; this version posted January 9, 2020. The copyright holder for this preprint (which was not certified by peer review) is the author/funder, who has granted bioRxiv a license to display the preprint in perpetuity. It is

B. fragilis NT

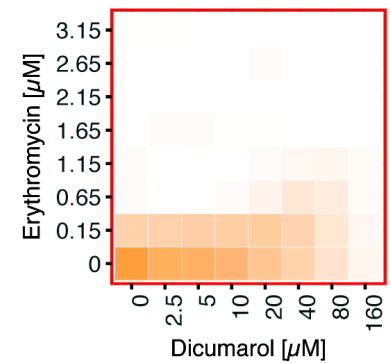

B. caccae

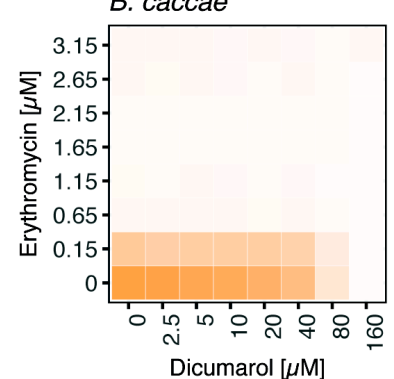

b

B. fragilis NT

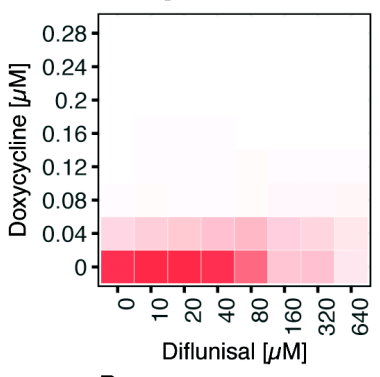

B. caccae

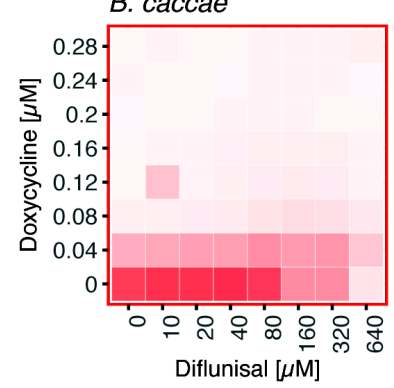

c

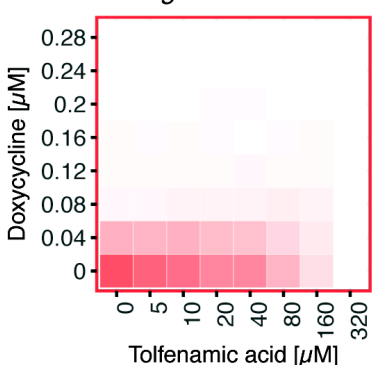

B. caccae

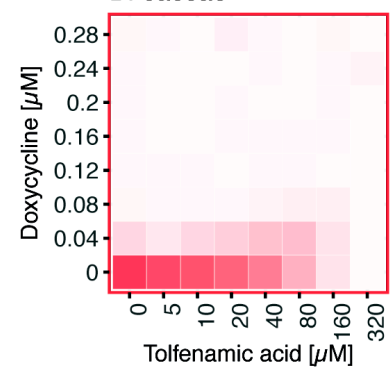

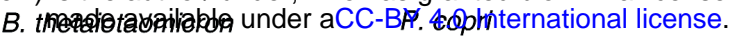

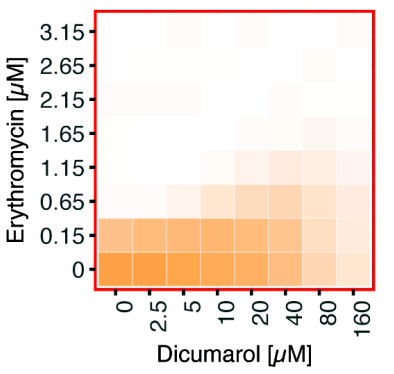

B. ovatus

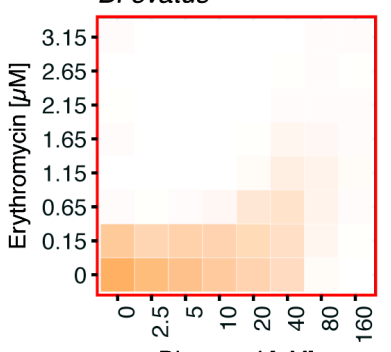

Dicumarol $[\mu \mathrm{M}]$

B. thetaiotaomicron

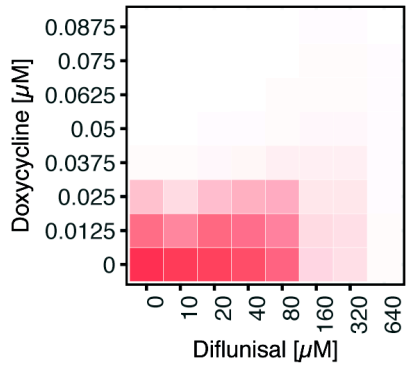

B. ovatus

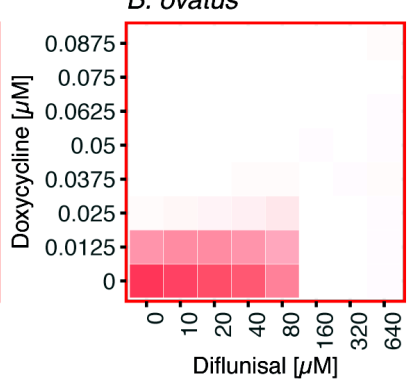

B. thetaiotaomicron

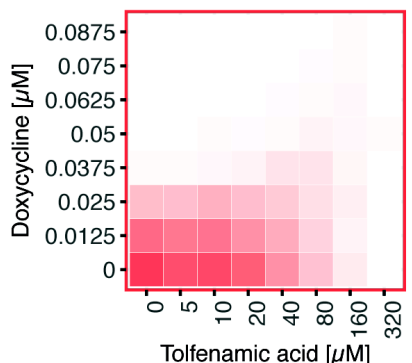

B. ovatus

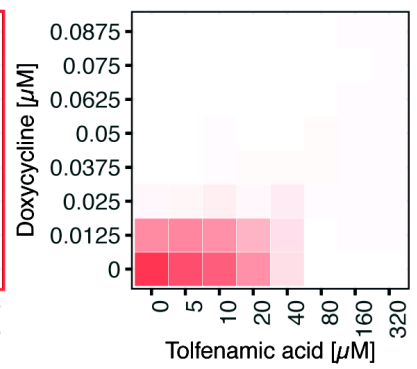

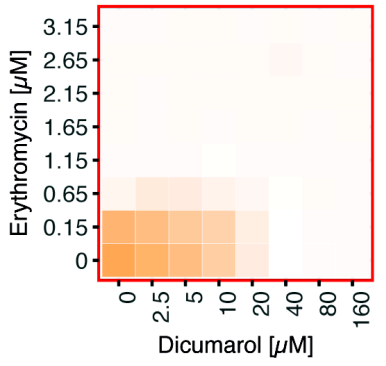

P. distasonis

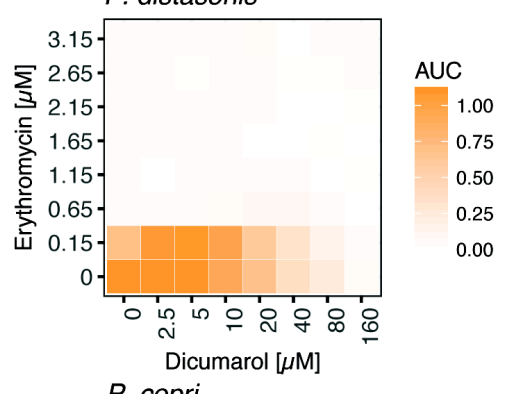

P. copri

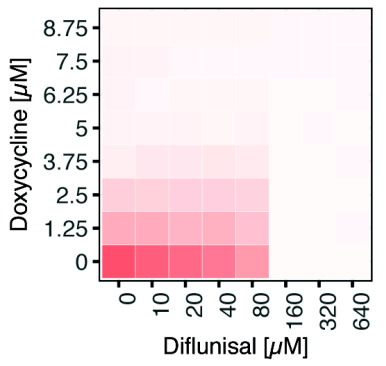

P. distasonis

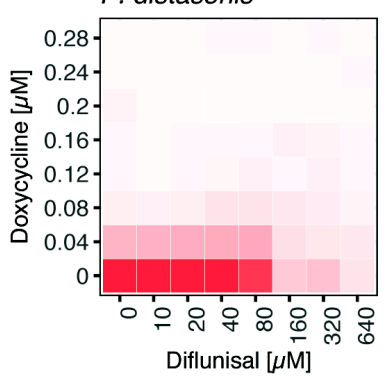

P. copri

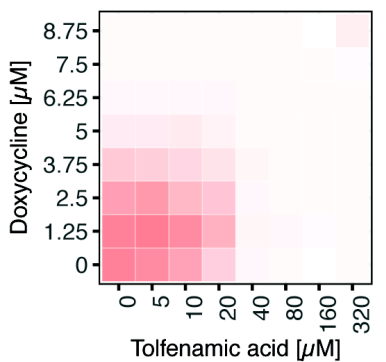

P. distasonis

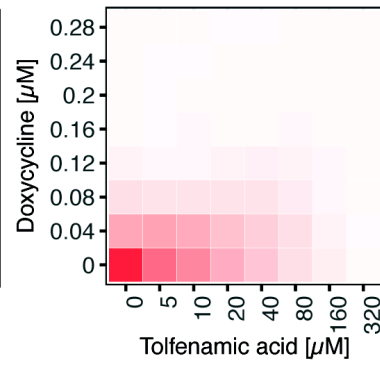

AUC

$-1.00$

0.75

0.50

0.25

0.00

\section{ED Figure 11}

Extended Data Figure 11 - Effect of antidotes on further gut commensals

$8 \times 8$ checkerboard assays to investigate if antidote is also protective for additional gut commensals for the following combinations: erythromycin and dicumarol (a), doxycycline and diflunisal (b) and doxycycline and tolfenamic acid (c). Heat map depicts bacterial growth based on median AUCs from two independent replicates. Red contours indicate antagonistic drug interactions. 
bioRxiv preprint doi: https://doi.org/10.1101/2020.01.09.893560; this version posted January 9, 2020. The copyright holder for this preprint (which was not certified by peer review) is the author/funder, who has granted bioRxiv a license to display the preprint in perpetuity. It is made available under aCC-BY 4.0 International license.

a
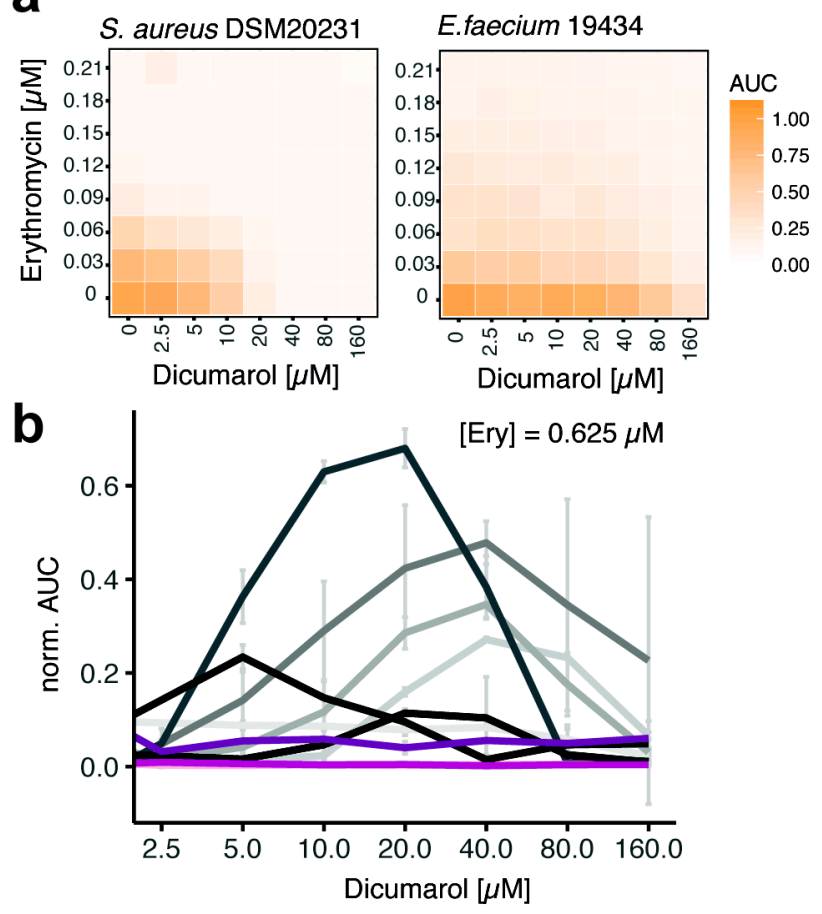

Commensal species Pathogenic species

$\begin{array}{ll}\text { B. caccae } & \text { E. faecium ATCC } 19434[1.25 \mu \mathrm{M}] \\ \text { B. fragilis NT } & \text { S. aureus DSM20231 [0.625 } \mu \mathrm{M}] \\ \text { B. ovatus } & \text { S. pneumoniae D39 [0.2 } \mu \mathrm{M}]\end{array}$

B. thetaiotaomicron

- B. vulgatus

- P. copri

- P. distasonis

\section{ED Figure 12}

Extended Data Figure 12- Effect of the antidote dicumarol on pathogens, relatively to commensal species.

a. Checkerboard assays for the drug combinations erythromycin-tolfenamic acid and erythromycin-dicumarol on the pathogens $S$. aureus (two different strains) and E. faecium. Heat map depict median normalized AUCs of checkerboard assays (at least three independent replicates).

b. Dicumarol rescues commensal growth (based on median AUCs, $\mathrm{N}=2$ ) at clinical relevant erythromycin concentrations in a concentrationdependent manner. Erythromycin still retains its activity against pertinent pathogens such as $S$. aureus, E. faecium and $S$. pneumoniae and is even slightly more active (synergy) for E. faecium (based on median AUCs, N=3). Error bars depict standard deviation. 